\title{
An iterative method for a common solution of generalized mixed equilibrium problems, variational inequalities, and hierarchical fixed point problems
}

\author{
Abdellah Bnouhachem ${ }^{1,2^{*}}$ and Ying Chen ${ }^{1}$
}

"Correspondence:

babedallah@yahoo.com

${ }^{1}$ School of Management Science

and Engineering, Nanjing University, Nanjing, 210093, P.R. China

2ENSA, Ibn Zohr University, Agadir,

1136, Morocco

\begin{abstract}
In this paper, we suggest and analyze an iterative method for finding a common solution of a variational inequality, a generalized mixed equilibrium problem, and a hierarchical fixed point problem in the setting of real Hilbert spaces. We prove the strong convergence of the sequence generated by the proposed method to a common solution of a variational inequality, a generalized mixed equilibrium problem, and a hierarchical fixed point problem. Several special cases are also discussed. The results presented in this paper extend and improve some well-known results in the literature.
\end{abstract}

MSC: 49J30; 47H09; 47J2O

Keywords: variational inequalities; generalized mixed problem; hierarchical fixed point problem; fixed point problem; projection method

\section{Introduction}

Let $H$ be a real Hilbert space whose inner product and norm are denoted by $\langle\cdot, \cdot\rangle$ and $\|\cdot\|$. Let $C$ be a nonempty closed convex subset of $H$. Let $F_{1}: C \times C \rightarrow \mathbb{R}$ be a bifunction, $D: C \rightarrow H$ be a nonlinear mapping, and $\varphi: C \rightarrow \mathbb{R}$ be a function. Recently, Peng and Yao [1] considered the following generalized mixed equilibrium problem (GMEP), which involves finding $x \in C$ such that

$$
F_{1}(x, y)+\varphi(y)-\varphi(x)+\langle D x, y-x\rangle \geq 0, \quad \forall y \in C .
$$

The set of solutions of $(1.1)$ is denoted by $\operatorname{GMEP}(F, \varphi, D)$. The GMEP is very general in the sense that it includes, as special cases, optimization problems, variational inequalities, minimax problems, and Nash equilibrium problems; see, for example, [2-5]. For instance, we quote reference [6] for a general system of generalized equilibrium problems.

Very recently, based on Yamada's hybrid steepest-descent method [7] and Colao, Marino, and Xu's hybrid viscosity approximation method [8], Ceng et al. [5] introduced a hybrid iterative method for finding a common element of the set of solutions of a generalized mixed equilibrium problem and the set of fixed points of finitely many nonexpansive

(c2014 Bnouhachem and Chen; licensee Springer. This is an Open Access article distributed under the terms of the Creative Commons Attribution License (http://creativecommons.org/licenses/by/2.0), which permits unrestricted use, distribution, and reproduction in any medium, provided the original work is properly cited. 
mappings in a real Hilbert space. Under suitable assumptions, they proved the strong iterative algorithm to a common solution of problem (1.1) and the fixed point problem of finitely many nonexpansive mappings. By combining Korpelevič's extragradient method [9], the hybrid steepest-descent method in [7], the viscosity approximation method, and the averaged mapping approach to the gradient-projection algorithm in [10], Al-Mazrooei et al. [2] proposed implicit and explicit iterative algorithms for finding a common element of the set of solutions of the convex minimization problem, the set of solutions of a finite family of generalized mixed equilibrium problems, and the set of solutions of a finite family of variational inequality problems for inverse strong monotone mappings in a real Hilbert space. Under very mild control conditions, they proved that the sequences generated by the proposed algorithms converge strongly to a common element of three sets, which is the unique solution of a variational inequality defined over the intersection of three sets.

If $B=0$, then the generalized mixed equilibrium problem (1.1) becomes the following mixed equilibrium problem: Find $x \in C$ such that

$$
F_{1}(x, y)+\varphi(y)-\varphi(x) \geq 0, \quad \forall y \in C .
$$

Problem (1.2) was studied by Ceng and Yao [11]. The set of solutions of (1.2) is denoted by $\operatorname{MEP}(F, \varphi, D)$.

If $\varphi=0$, then the generalized mixed equilibrium problem (1.1) becomes the following generalized equilibrium problem: Find $x \in C$ such that

$$
F_{1}(x, y)+\langle D x, y-x\rangle \geq 0, \quad \forall y \in C .
$$

Problem (1.3) was studied by Takahashi and Takahashi [12]. The set of solutions of (1.3) is denoted by $\operatorname{GEP}(F, D)$.

If $\varphi=0$ and $B=0$, then the generalized mixed equilibrium problem (1.1) becomes the following equilibrium problem: Find $x \in C$ such that

$$
F_{1}(x, y) \geq 0, \quad \forall y \in C
$$

The solution set of (1.4) is denoted by $E P(F)$. Numerous problems in physics, optimization, and economics reduce to finding a solution of (1.4), see [13, 14].

Let $A$ be a mapping from $C$ into $H$. A classical variational inequality problem is to find a vector $u \in C$ such that

$$
\langle v-u, A u\rangle \geq 0, \quad \forall v \in C
$$

The solution set of (1.5) is denoted by $V I(C, A)$. It is easy to observe that

$$
u^{*} \in V I(C, A) \quad \Longleftrightarrow \quad u^{*}=P_{C}\left[u^{*}-\rho A u^{*}\right], \quad \text { where } \rho>0 .
$$

We now have a variety of techniques to suggest and analyze various iterative algorithms for solving variational inequalities and related optimization problems; see [1-41]. The fixed point theory has played an important role in the development of various algorithms for 
solving variational inequalities. Using the projection operator technique, one usually establishes an equivalence between variational inequalities and fixed point problems. We introduce the following definitions, which are useful in the following analysis.

Definition 1.1 The mapping $T: C \rightarrow H$ is said to be

(a) monotone if

$$
\langle T x-T y, x-y\rangle \geq 0, \quad \forall x, y \in C
$$

(b) strongly monotone if there exists $\alpha>0$ such that

$$
\langle T x-T y, x-y\rangle \geq \alpha\|x-y\|^{2}, \quad \forall x, y \in C ;
$$

(c) $\alpha$-inverse strongly monotone if there exists $\alpha>0$ such that

$$
\langle T x-T y, x-y\rangle \geq \alpha\|T x-T y\|^{2}, \quad \forall x, y \in C ;
$$

(d) nonexpansive if

$$
\|T x-T y\| \leq\|x-y\|, \quad \forall x, y \in C
$$

(e) $k$-Lipschitz continuous if there exists a constant $k>0$ such that

$$
\|T x-T y\| \leq k\|x-y\|, \quad \forall x, y \in C ;
$$

(f) a contraction on $C$ if there exists a constant $0 \leq k<1$ such that

$$
\|T x-T y\| \leq k\|x-y\|, \quad \forall x, y \in C .
$$

It is easy to observe that every $\alpha$-inverse strongly monotone $T$ is monotone and Lipschitz continuous. It is well known that every nonexpansive operator $T: H \rightarrow H$ satisfies, for all $(x, y) \in H \times H$, the inequality

$$
\langle(x-T(x))-(y-T(y)), T(y)-T(x)\rangle \leq \frac{1}{2}\|(T(x)-x)-(T(y)-y)\|^{2}
$$

and therefore, we get, for all $(x, y) \in H \times \operatorname{Fix}(T)$,

$$
\langle x-T(x), y-T(x)\rangle \leq \frac{1}{2}\|T(x)-x\|^{2} .
$$

The fixed point problem for the mapping $T$ is to find $x \in C$ such that

$$
T x=x
$$

We denote by $F(T)$ the set of solutions of (1.8). It is well known that $F(T)$ is closed and convex, and $P_{F}(T)$ is well defined. 
Let $S: C \rightarrow H$ be a nonexpansive mapping. The following problem is called a hierarchical fixed point problem: Find $x \in F(T)$ such that

$$
\langle x-S x, y-x\rangle \geq 0, \quad \forall y \in F(T)
$$

It is known that the hierarchical fixed point problem (1.9) links with some monotone variational inequalities and convex programming problems; see [15]. Various methods have been proposed to solve the hierarchical fixed point problem; see [16-20]. In 2010, Yao et al. [15] introduced the following strong convergence iterative algorithm to solve problem (1.9):

$$
\begin{aligned}
& y_{n}=\beta_{n} S x_{n}+\left(1-\beta_{n}\right) x_{n}, \\
& x_{n+1}=P_{C}\left[\alpha_{n} f\left(x_{n}\right)+\left(1-\alpha_{n}\right) T y_{n}\right], \quad \forall n \geq 0,
\end{aligned}
$$

where $f: C \rightarrow H$ is a contraction mapping and $\left\{\alpha_{n}\right\}$ and $\left\{\beta_{n}\right\}$ are two sequences in $(0,1)$. Under some certain restrictions on the parameters, Yao et al. proved that the sequence $\left\{x_{n}\right\}$ generated by (1.10) converges strongly to $z \in F(T)$, which is the unique solution of the following variational inequality:

$$
\langle(I-f) z, y-z\rangle \geq 0, \quad \forall y \in F(T)
$$

In 2011, Ceng et al. [21] investigated the following iterative method:

$$
x_{n+1}=P_{C}\left[\alpha_{n} \rho U\left(x_{n}\right)+\left(I-\alpha_{n} \mu F\right)\left(T\left(y_{n}\right)\right)\right], \quad \forall n \geq 0,
$$

where $U$ is a Lipschitzian mapping, and $F$ is a Lipschitzian and strongly monotone mapping. They proved that under some approximate assumptions on the operators and parameters, the sequence $\left\{x_{n}\right\}$ generated by (1.12) converges strongly to the unique solution of the variational inequality

$$
\langle\rho U(z)-\mu F(z), x-z\rangle \geq 0, \quad \forall x \in \operatorname{Fix}(T)
$$

Very recently, Ceng et al. [22] introduced and analyzed hybrid implicit and explicit viscosity iterative algorithms for solving a general system of variational inequalities with hierarchical fixed point problem constraint for a countable family of nonexpansive mappings in a real Banach space, which can be viewed as an extension and improvement of the recent results in the literature.

In this paper, motivated by the work of Ceng et al. [5, 21, 24], Al-Mazrooei et al. [2], Yao et al. [15], Bnouhachem [23] and by the recent work going in this direction, we give an iterative method for finding the approximate element of the common set of solutions of (1.1), (1.5), and (1.9) in a real Hilbert space. We establish a strong convergence theorem based on this method. We would like to mention that our proposed method is quite general and flexible and includes many known results for solving of variational inequality problems, mixed equilibrium problems and hierarchical fixed point problems; see, e.g., [15, 16, 18, 21, $23,25]$ and relevant references cited therein. 


\section{Preliminaries}

In this section, we list some fundamental lemmas that are useful in the consequent analysis. The first lemma provides some basic properties of projection onto $C$.

Lemma 2.1 Let $P_{C}$ denote the projection of $H$ onto $C$. Then we have the following inequalities:

$$
\begin{aligned}
& \left\langle z-P_{C}[z], P_{C}[z]-v\right\rangle \geq 0, \quad \forall z \in H, v \in C ; \\
& \left\langle u-v, P_{C}[u]-P_{C}[v]\right\rangle \geq\left\|P_{C}[u]-P_{C}[v]\right\|^{2}, \quad \forall u, v \in H ; \\
& \left\|P_{C}[u]-P_{C}[v]\right\| \leq\|u-v\|, \quad \forall u, v \in H ; \\
& \left\|u-P_{C}[z]\right\|^{2} \leq\|z-u\|^{2}-\left\|z-P_{C}[z]\right\|^{2}, \quad \forall z \in H, u \in C .
\end{aligned}
$$

Assumption 2.1 [1] Let $F_{1}: C \times C \rightarrow \mathbb{R}$ be a bifunction and $\varphi: C \rightarrow \mathbb{R}$ be a function satisfying the following assumptions:

$\left(\mathrm{A}_{1}\right) F_{1}(x, x)=0, \forall x \in C$;

$\left(\mathrm{A}_{2}\right) F_{1}$ is monotone, i.e., $F_{1}(x, y)+F_{1}(y, x) \leq 0, \forall x, y \in C$;

$\left(\mathrm{A}_{3}\right)$ for each $x, y, z \in C, \lim _{t \rightarrow 0} F_{1}(t z+(1-t) x, y) \leq F_{1}(x, y)$;

$\left(\mathrm{A}_{4}\right)$ for each $x \in C, y \rightarrow F_{1}(x, y)$ is convex and lower semicontinuous;

$\left(\mathrm{B}_{1}\right)$ for each $x \in H$ and $r>0$, there exists a bounded subset $K$ of $C$ and $y_{x} \in C \cap \operatorname{dom}(\varphi)$ such that

$$
F_{1}\left(z, y_{x}\right)+\varphi\left(y_{x}\right)-\varphi(z)+\frac{1}{r}\left\langle y_{x}-z, z-x\right\rangle<0, \quad \forall z \in C \backslash K ;
$$

$\left(\mathrm{B}_{2}\right) \quad C$ is a bounded set.

Lemma 2.2 [1] Let $C$ be a nonempty closed convex subset of $H$. Let $F_{1}: C \times C \rightarrow \mathbb{R}$ satisfy $\left(\mathrm{A}_{1}\right)-\left(\mathrm{A}_{3}\right)$, and let $\varphi: C \rightarrow \mathbb{R}$ be a proper lower semicontinuous and convex function. Assume that either $\left(\mathrm{B}_{1}\right)$ or $\left(\mathrm{B}_{2}\right)$ holds. For $r>0$ and $\forall x \in H$, define a mapping $T_{r}: H \rightarrow C$ as follows:

$$
T_{r}(x)=\left\{z \in C: F_{1}(z, y)+\varphi(y)-\varphi(z)+\frac{1}{r}\langle y-z, z-x\rangle \geq 0, \forall y \in C\right\} .
$$

Then the following hold:

(i) $T_{r}$ is nonempty and single-valued;

(ii) $T_{r}$ is firmly nonexpansive, i.e.,

$$
\left\|T_{r}(x)-T_{r}(y)\right\|^{2} \leq\left\langle T_{r}(x)-T_{r}(y), x-y\right\rangle, \quad \forall x, y \in H
$$

(iii) $F\left(T_{r}(I-r D)\right)=G M E P(F, \varphi, D)$;

(iv) $\operatorname{GMEP}(F, \varphi, D)$ is closed and convex.

Lemma 2.3 [26] Let $C$ be a nonempty closed convex subset of a real Hilbert space H. If $T$ : $C \rightarrow C$ is a nonexpansive mapping with $\operatorname{Fix}(T) \neq \emptyset$, then the mapping $I-T$ is demiclosed at 0 , i.e., if $\left\{x_{n}\right\}$ is a sequence in $C$ weakly converging to $x$ and if $\left\{(I-T) x_{n}\right\}$ converges strongly to 0 , then $(I-T) x=0$. 
Lemma 2.4 [21] Let $U: C \rightarrow H$ be a $\tau$-Lipschitzian mapping, and let $F: C \rightarrow H$ be a $k$ Lipschitzian and $\eta$-strongly monotone mapping, then for $0 \leq \rho \tau<\mu \eta, \mu F-\rho U$ is $\mu \eta-\rho \tau$ strongly monotone, i.e.,

$$
\langle(\mu F-\rho U) x-(\mu F-\rho U) y, x-y\rangle \geq(\mu \eta-\rho \tau)\|x-y\|^{2}, \quad \forall x, y \in C .
$$

Lemma 2.5 [27] Suppose that $\lambda \in(0,1)$ and $\mu>0$. Let $F: C \rightarrow H$ be a $k$-Lipschitzian and $\eta$-strongly monotone operator. In association with a nonexpansive mapping $T: C \rightarrow C$, define the mapping $T^{\lambda}: \mathrm{C} \rightarrow \mathrm{H}$ by

$$
T^{\lambda} x=T x-\lambda \mu F T(x), \quad \forall x \in C .
$$

Then $T^{\lambda}$ is a contraction provided $\mu<\frac{2 \eta}{k^{2}}$, that is,

$$
\left\|T^{\lambda} x-T^{\lambda} y\right\| \leq(1-\lambda \nu)\|x-y\|, \quad \forall x, y \in C,
$$

where $v=1-\sqrt{1-\mu\left(2 \eta-\mu k^{2}\right)}$.

Lemma 2.6 [28] Assume that $\left\{a_{n}\right\}$ is a sequence of nonnegative real numbers such that

$$
a_{n+1} \leq\left(1-v_{n}\right) a_{n}+\delta_{n}
$$

where $\left\{v_{n}\right\}$ is a sequence in $(0,1)$ and $\delta_{n}$ is a sequence such that

(1) $\sum_{n=1}^{\infty} v_{n}=\infty$;

(2) $\lim \sup _{n \rightarrow \infty} \delta_{n} / v_{n} \leq 0$ or $\sum_{n=1}^{\infty}\left|\delta_{n}\right|<\infty$.

Then $\lim _{n \rightarrow \infty} a_{n}=0$.

Lemma 2.7 [29] Let C be a closed convex subset of $H$. Let $\left\{x_{n}\right\}$ be a bounded sequence in $H$. Assume that

(i) the weak $w$-limit set $w_{w}\left(x_{n}\right) \subset C$, where $w_{w}\left(x_{n}\right)=\left\{x: x_{n_{i}} \rightarrow x\right\}$;

(ii) for each $z \in C, \lim _{n \rightarrow \infty}\left\|x_{n}-z\right\|$ exists.

Then $\left\{x_{n}\right\}$ is weakly convergent to a point in $C$.

\section{The proposed method and some properties}

In this section, we suggest and analyze our method for finding common solutions of the generalized mixed equilibrium problem (1.1), the variational inequality (1.5), and the hierarchical fixed point problem (1.9).

Let $C$ be a nonempty closed convex subset of a real Hilbert space $H$. Let $D, A: C \rightarrow H$ be $\theta, \alpha$-inverse strongly monotone mappings, respectively. Let $F_{1}: C \times C \rightarrow \mathbb{R}$ satisfy $\left(\mathrm{A}_{1}\right)$ $\left(\mathrm{A}_{4}\right)$, and let $\varphi: C \rightarrow \mathbb{R}$ be a proper lower semicontinuous and convex function. Let $S, T:$ $C \rightarrow C$ be nonexpansive mappings such that $F(T) \cap \operatorname{VI}(C, A) \cap \operatorname{GMEP}(F, \varphi, D) \neq \emptyset$. Let $F: C \rightarrow C$ be a $k$-Lipschitzian mapping and be $\eta$-strongly monotone, and let $U: C \rightarrow C$ be a $\tau$-Lipschitzian mapping. 
Algorithm 3.1 For an arbitrarily given $x_{0} \in C$, let the iterative sequences $\left\{u_{n}\right\},\left\{x_{n}\right\},\left\{y_{n}\right\}$, and $\left\{z_{n}\right\}$ be generated by

$$
\left\{\begin{array}{l}
F_{1}\left(u_{n}, y\right)+\left\langle D x_{n}, y-u_{n}\right\rangle+\varphi(y)-\varphi\left(u_{n}\right)+\frac{1}{r_{n}}\left\langle y-u_{n}, u_{n}-x_{n}\right\rangle \geq 0, \quad \forall y \in C ; \\
z_{n}=P_{C}\left[u_{n}-\lambda_{n} A u_{n}\right] ; \\
y_{n}=\beta_{n} S x_{n}+\left(1-\beta_{n}\right) z_{n} ; \\
x_{n+1}=P_{C}\left[\alpha_{n} \rho U\left(x_{n}\right)+\gamma_{n} x_{n}+\left(\left(1-\gamma_{n}\right) I-\alpha_{n} \mu F\right)\left(T\left(y_{n}\right)\right)\right], \quad \forall n \geq 0,
\end{array}\right.
$$

where $\lambda_{n} \in(0,2 \alpha),\left\{r_{n}\right\} \subset(0,2 \theta)$. Suppose that the parameters satisfy $0<\mu<\frac{2 \eta}{k^{2}}, 0 \leq \rho \tau<$ $\nu$, where $v=1-\sqrt{1-\mu\left(2 \eta-\mu k^{2}\right)}$. Also $\left\{\gamma_{n}\right\},\left\{\alpha_{n}\right\}$, and $\left\{\beta_{n}\right\}$ are sequences in $(0,1)$ satisfying the following conditions:

(a) $\lim _{n \rightarrow \infty} \gamma_{n}=0, \gamma_{n}+\alpha_{n}<1$,

(b) $\lim _{n \rightarrow \infty} \alpha_{n}=0$ and $\sum_{n=1}^{\infty} \alpha_{n}=\infty$,

(c) $\lim _{n \rightarrow \infty}\left(\beta_{n} / \alpha_{n}\right)=0$,

(d) $\sum_{n=1}^{\infty}\left|\alpha_{n-1}-\alpha_{n}\right|<\infty, \sum_{n=1}^{\infty}\left|\gamma_{n-1}-\gamma_{n}\right|<\infty$, and $\sum_{n=1}^{\infty}\left|\beta_{n-1}-\beta_{n}\right|<\infty$,

(e) $\liminf _{n \rightarrow \infty} r_{n}>0$ and $\sum_{n=1}^{\infty}\left|r_{n-1}-r_{n}\right|<\infty$,

(f) $\liminf _{n \rightarrow \infty} \lambda_{n}<\limsup \sup _{n \rightarrow \infty} \lambda_{n}<2 \alpha$ and $\sum_{n=1}^{\infty}\left|\lambda_{n-1}-\lambda_{n}\right|<\infty$.

Remark 3.1 Our method can be viewed as an extension and improvement for some wellknown results, for example, the following.

- If $\gamma_{n}=0$, the proposed method is an extension and improvement of the method of Bnouhachem [23] and Wang and $\mathrm{Xu}$ [30] for finding the approximate element of the common set of solutions of generalized mixed equilibrium and hierarchical fixed point problems in a real Hilbert space.

- If we have the Lipschitzian mapping $U=f, F=I, \rho=\mu=1, \gamma_{n}=0$, and $A=0$, we obtain an extension and improvement of the method of Yao et al. [15] for finding the approximate element of the common set of solutions of generalized mixed equilibrium and hierarchical fixed point problems in a real Hilbert space.

- The contractive mapping $f$ with a coefficient $\alpha \in[0,1)$ in other papers $[15,25,27]$ is extended to the cases of the Lipschitzian mapping $U$ with a coefficient constant $\gamma \in[0, \infty)$.

This shows that Algorithm 3.1 is quite general and unifying.

Lemma 3.1 Let $x^{*} \in F(T) \cap V I(C, D) \cap \operatorname{GMEP}(F, \varphi, D)$. Then $\left\{x_{n}\right\},\left\{u_{n}\right\},\left\{z_{n}\right\}$, and $\left\{y_{n}\right\}$ are bounded.

Proof First, we show that the mapping $\left(I-r_{n} D\right)$ is nonexpansive. For any $x, y \in C$,

$$
\begin{aligned}
\left\|\left(I-r_{n} D\right) x-\left(I-r_{n} D\right) y\right\|^{2} & =\left\|(x-y)-r_{n}(D x-D y)\right\|^{2} \\
& =\|x-y\|^{2}-2 r_{n}\langle x-y, D x-D y\rangle+r_{n}^{2}\|D x-D y\|^{2} \\
& \leq\|x-y\|^{2}-r_{n}\left(2 \theta-r_{n}\right)\|D x-D y\|^{2} \\
& \leq\|x-y\|^{2} .
\end{aligned}
$$

Similarly, we can show that the mapping $\left(I-\lambda_{n} A\right)$ is nonexpansive. It follows from Lemma 2.2 that $u_{n}=T_{r_{n}}\left(x_{n}-r_{n} D x_{n}\right)$. Let $x^{*} \in F(T) \cap V I(C, D) \cap \operatorname{GMEP}(F, \varphi, D)$, we have 


$$
\begin{aligned}
& x^{*}=T_{r_{n}}\left(x^{*}-r_{n} D x^{*}\right) . \\
& \left\|u_{n}-x^{*}\right\|^{2}=\left\|T_{r_{n}}\left(x_{n}-r_{n} D x_{n}\right)-T_{r_{n}}\left(x^{*}-r_{n} D x^{*}\right)\right\|^{2} \\
& \leq\left\|\left(x_{n}-r_{n} D x_{n}\right)-\left(x^{*}-r_{n} D x^{*}\right)\right\|^{2} \\
& \leq\left\|x_{n}-x^{*}\right\|^{2}-r_{n}\left(2 \theta-r_{n}\right)\left\|D x_{n}-D x^{*}\right\|^{2} \\
& \leq\left\|x_{n}-x^{*}\right\|^{2} \text {. }
\end{aligned}
$$

Since the mapping $A$ is $\alpha$-inverse strongly monotone, we have

$$
\begin{aligned}
\left\|z_{n}-x^{*}\right\|^{2} & =\left\|P_{C}\left[u_{n}-\lambda_{n} A u_{n}\right]-P_{C}\left[x^{*}-\lambda_{n} A x^{*}\right]\right\|^{2} \\
& \leq\left\|u_{n}-x^{*}-\lambda_{n}\left(A u_{n}-A x^{*}\right)\right\|^{2} \\
& \leq\left\|u_{n}-x^{*}\right\|^{2}-\lambda_{n}\left(2 \alpha-\lambda_{n}\right)\left\|A u_{n}-A x^{*}\right\|^{2} \\
& \leq\left\|u_{n}-x^{*}\right\|^{2} \\
& \leq\left\|x_{n}-x^{*}\right\|^{2} .
\end{aligned}
$$

We define $V_{n}=\alpha_{n} \rho U\left(x_{n}\right)+\gamma_{n} x_{n}+\left(\left(1-\gamma_{n}\right) I-\alpha_{n} \mu F\right)\left(T\left(y_{n}\right)\right)$. Next, we prove that the sequence $\left\{x_{n}\right\}$ is bounded, and without loss of generality we can assume that $\beta_{n} \leq \alpha_{n}$ for all $n \geq 1$. From (3.1), we have

$$
\begin{aligned}
& \left\|x_{n+1}-x^{*}\right\|=\left\|P_{C}\left[V_{n}\right]-P_{C}\left[x^{*}\right]\right\| \\
& \leq\left\|\alpha_{n} \rho U\left(x_{n}\right)+\gamma_{n} x_{n}+\left(\left(1-\gamma_{n}\right) I-\alpha_{n} \mu F\right)\left(T\left(y_{n}\right)\right)-x^{*}\right\| \\
& =\| \alpha_{n}\left(\rho U\left(x_{n}\right)-\mu F\left(x^{*}\right)\right)+\gamma_{n}\left(x_{n}-x^{*}\right)+\left(\left(1-\gamma_{n}\right) I-\alpha_{n} \mu F\right)\left(T\left(y_{n}\right)\right) \\
& -\left(\left(1-\gamma_{n}\right) I-\alpha_{n} \mu F\right)\left(T\left(x^{*}\right)\right) \| \\
& \leq \alpha_{n}\left\|\rho U\left(x_{n}\right)-\mu F\left(x^{*}\right)\right\|+\gamma_{n}\left\|x_{n}-x^{*}\right\| \\
& +\left(1-\gamma_{n}\right)\left\|\left(I-\frac{\alpha_{n} \mu}{1-\gamma_{n}} F\right)\left(T\left(y_{n}\right)\right)-\left(I-\frac{\alpha_{n} \mu}{1-\gamma_{n}} F\right) T\left(x^{*}\right)\right\| \\
& =\alpha_{n}\left\|\rho U\left(x_{n}\right)-\rho U\left(x^{*}\right)+(\rho U-\mu F) x^{*}\right\|+\gamma_{n}\left\|x_{n}-x^{*}\right\| \\
& +\left(1-\gamma_{n}\right)\left\|\left(I-\frac{\alpha_{n} \mu}{1-\gamma_{n}} F\right)\left(T\left(y_{n}\right)\right)-\left(I-\frac{\alpha_{n} \mu}{1-\gamma_{n}} F\right) T\left(x^{*}\right)\right\| \\
& \leq \alpha_{n} \rho \tau\left\|x_{n}-x^{*}\right\|+\alpha_{n}\left\|(\rho U-\mu F) x^{*}\right\|+\gamma_{n}\left\|x_{n}-x^{*}\right\| \\
& +\left(1-\gamma_{n}\right)\left(1-\frac{\alpha_{n} v}{1-\gamma_{n}}\right)\left\|y_{n}-x^{*}\right\| \\
& \leq \alpha_{n} \rho \tau\left\|x_{n}-x^{*}\right\|+\alpha_{n}\left\|(\rho U-\mu F) x^{*}\right\|+\gamma_{n}\left\|x_{n}-x^{*}\right\| \\
& +\left(1-\gamma_{n}-\alpha_{n} \nu\right)\left\|\beta_{n} S x_{n}+\left(1-\beta_{n}\right) z_{n}-x^{*}\right\| \\
& \leq \alpha_{n} \rho \tau\left\|x_{n}-x^{*}\right\|+\alpha_{n}\left\|(\rho U-\mu F) x^{*}\right\|+\gamma_{n}\left\|x_{n}-x^{*}\right\| \\
& +\left(1-\gamma_{n}-\alpha_{n} \nu\right)\left(\beta_{n}\left\|S x_{n}-S x^{*}\right\|+\beta_{n}\left\|S x^{*}-x^{*}\right\|+\left(1-\beta_{n}\right)\left\|z_{n}-x^{*}\right\|\right) \\
& \leq \alpha_{n} \rho \tau\left\|x_{n}-x^{*}\right\|+\alpha_{n}\left\|(\rho U-\mu F) x^{*}\right\|+\gamma_{n}\left\|x_{n}-x^{*}\right\| \\
& +\left(1-\gamma_{n}-\alpha_{n} v\right)\left(\beta_{n}\left\|S x_{n}-S x^{*}\right\|+\beta_{n}\left\|S x^{*}-x^{*}\right\|+\left(1-\beta_{n}\right)\left\|x_{n}-x^{*}\right\|\right)
\end{aligned}
$$




$$
\begin{aligned}
\leq & \alpha_{n} \rho \tau\left\|x_{n}-x^{*}\right\|+\alpha_{n}\left\|(\rho U-\mu F) x^{*}\right\|+\gamma_{n}\left\|x_{n}-x^{*}\right\| \\
& +\left(1-\gamma_{n}-\alpha_{n} \nu\right)\left(\beta_{n}\left\|x_{n}-x^{*}\right\|+\beta_{n}\left\|S x^{*}-x^{*}\right\|+\left(1-\beta_{n}\right)\left\|x_{n}-x^{*}\right\|\right) \\
= & \left(1-\alpha_{n}(v-\rho \tau)\right)\left\|x_{n}-x^{*}\right\|+\alpha_{n}\left\|(\rho U-\mu F) x^{*}\right\| \\
& +\left(1-\gamma_{n}-\alpha_{n} v\right) \beta_{n}\left\|S x^{*}-x^{*}\right\| \\
\leq & \left(1-\alpha_{n}(v-\rho \tau)\right)\left\|x_{n}-x^{*}\right\|+\alpha_{n}\left\|(\rho U-\mu F) x^{*}\right\|+\beta_{n}\left\|S x^{*}-x^{*}\right\| \\
\leq & \left(1-\alpha_{n}(v-\rho \tau)\right)\left\|x_{n}-x^{*}\right\|+\alpha_{n}\left(\left\|(\rho U-\mu F) x^{*}\right\|+\left\|S x^{*}-x^{*}\right\|\right) \\
= & \left(1-\alpha_{n}(v-\rho \tau)\right)\left\|x_{n}-x^{*}\right\|+\frac{\alpha_{n}(v-\rho \tau)}{v-\rho \tau}\left(\left\|(\rho U-\mu F) x^{*}\right\|+\left\|S x^{*}-x^{*}\right\|\right) \\
\leq & \max \left\{\left\|x_{n}-x^{*}\right\|, \frac{1}{v-\rho \tau}\left(\left\|(\rho U-\mu F) x^{*}\right\|+\left\|S x^{*}-x^{*}\right\|\right)\right\},
\end{aligned}
$$

where the third inequality follows from Lemma 2.5. By induction on $n$, we obtain $\| x_{n}-$ $x^{*} \| \leq \max \left\{\left\|x_{0}-x^{*}\right\|, \frac{1}{v-\rho \tau}\left(\left\|(\rho U-\mu F) x^{*}\right\|+\left\|S x^{*}-x^{*}\right\|\right)\right\}$ for $n \geq 0$ and $x_{0} \in C$. Hence $\left\{x_{n}\right\}$ is bounded, and consequently, we deduce that $\left\{u_{n}\right\},\left\{z_{n}\right\},\left\{v_{n}\right\},\left\{y_{n}\right\},\left\{S\left(x_{n}\right)\right\},\left\{T\left(x_{n}\right)\right\}$, $\left\{F\left(T\left(y_{n}\right)\right)\right\}$, and $\left\{U\left(x_{n}\right)\right\}$ are bounded.

Lemma 3.2 Let $x^{*} \in F(T) \cap \operatorname{VI}(C, D) \cap \operatorname{GMEP}(F, \varphi, D)$ and $\left\{x_{n}\right\}$ be the sequence generated by Algorithm 3.1. Then we have

(a) $\lim _{n \rightarrow \infty}\left\|x_{n+1}-x_{n}\right\|=0$.

(b) The weak $w$-limit set $w_{w}\left(x_{n}\right) \subset F(T)\left(w_{w}\left(x_{n}\right)=\left\{x: x_{n_{i}} \rightarrow x\right\}\right)$.

Proof From the nonexpansivity of the mapping $\left(I-\lambda_{n} A\right)$ and $P_{C}$, we have

$$
\begin{aligned}
\left\|z_{n}-z_{n-1}\right\| & \leq\left\|\left(u_{n}-\lambda_{n} A u_{n}\right)-\left(u_{n-1}-\lambda_{n-1} A u_{n-1}\right)\right\| \\
& =\left\|\left(u_{n}-u_{n-1}\right)-\lambda_{n}\left(A u_{n}-A u_{n-1}\right)-\left(\lambda_{n}-\lambda_{n-1}\right) A u_{n-1}\right\| \\
& \leq\left\|\left(u_{n}-u_{n-1}\right)-\lambda_{n}\left(A u_{n}-A u_{n-1}\right)\right\|+\left|\lambda_{n}-\lambda_{n-1}\right|\left\|A u_{n-1}\right\| \\
& \leq\left\|u_{n}-u_{n-1}\right\|+\left|\lambda_{n}-\lambda_{n-1}\right|\left\|A u_{n-1}\right\| .
\end{aligned}
$$

Next, we estimate that

$$
\begin{aligned}
& \left\|y_{n}-y_{n-1}\right\| \\
& \quad \leq\left\|\beta_{n} S x_{n}+\left(1-\beta_{n}\right) z_{n}-\left(\beta_{n-1} S x_{n-1}+\left(1-\beta_{n-1}\right) z_{n-1}\right)\right\| \\
& \quad=\left\|\beta_{n}\left(S x_{n}-S x_{n-1}\right)+\left(\beta_{n}-\beta_{n-1}\right) S x_{n-1}+\left(1-\beta_{n}\right)\left(z_{n}-z_{n-1}\right)+\left(\beta_{n-1}-\beta_{n}\right) z_{n-1}\right\| \\
& \quad \leq \beta_{n}\left\|x_{n}-x_{n-1}\right\|+\left(1-\beta_{n}\right)\left\|z_{n}-z_{n-1}\right\|+\left|\beta_{n}-\beta_{n-1}\right|\left(\left\|S x_{n-1}\right\|+\left\|z_{n-1}\right\|\right) .
\end{aligned}
$$

It follows from (3.3) and (3.4) that

$$
\begin{aligned}
\left\|y_{n}-y_{n-1}\right\| \leq & \beta_{n}\left\|x_{n}-x_{n-1}\right\|+\left(1-\beta_{n}\right)\left\{\left\|u_{n}-u_{n-1}\right\|+\left|\lambda_{n}-\lambda_{n-1}\right|\left\|A u_{n-1}\right\|\right\} \\
& +\left|\beta_{n}-\beta_{n-1}\right|\left(\left\|S x_{n-1}\right\|+\left\|z_{n-1}\right\|\right) .
\end{aligned}
$$


On the other hand, $u_{n}=T_{r_{n}}\left(x_{n}-r_{n} D x_{n}\right)$ and $u_{n-1}=T_{r_{n-1}}\left(x_{n-1}-r_{n-1} D x_{n-1}\right)$, we have

$$
F_{1}\left(u_{n}, y\right)+\varphi(y)-\varphi\left(u_{n}\right)+\left\langle D x_{n}, y-u_{n}\right\rangle+\frac{1}{r_{n}}\left\langle y-u_{n}, u_{n}-x_{n}\right\rangle \geq 0, \quad \forall y \in C
$$

and

$$
\begin{aligned}
& F_{1}\left(u_{n-1}, y\right)+\varphi(y)-\varphi\left(u_{n-1}\right)+\left\langle D x_{n-1}, y-u_{n-1}\right\rangle+\frac{1}{r_{n-1}}\left\langle y-u_{n-1}, u_{n-1}-x_{n-1}\right\rangle \geq 0, \\
& \quad \forall y \in C .
\end{aligned}
$$

Taking $y=u_{n-1}$ in (3.6) and $y=u_{n}$ in (3.7), we get

$$
F_{1}\left(u_{n}, u_{n-1}\right)+\varphi\left(u_{n-1}\right)-\varphi\left(u_{n}\right)+\left\langle D x_{n}, u_{n-1}-u_{n}\right\rangle+\frac{1}{r_{n}}\left\langle u_{n-1}-u_{n}, u_{n}-x_{n}\right\rangle \geq 0
$$

and

$$
\begin{gathered}
F_{1}\left(u_{n-1}, u_{n}\right)+\varphi\left(u_{n}\right)-\varphi\left(u_{n-1}\right)+\left\langle D x_{n-1}, u_{n}-u_{n-1}\right\rangle \\
+\frac{1}{r_{n-1}}\left\langle u_{n}-u_{n-1}, u_{n-1}-x_{n-1}\right\rangle \geq 0 .
\end{gathered}
$$

Adding (3.8) and (3.9) and using the monotonicity of $F_{1}$, we have

$$
\left\langle D x_{n-1}-D x_{n}, u_{n}-u_{n-1}\right\rangle+\left\langle u_{n}-u_{n-1}, \frac{u_{n-1}-x_{n-1}}{r_{n-1}}-\frac{u_{n}-x_{n}}{r_{n}}\right\rangle \geq 0,
$$

which implies that

$$
\begin{aligned}
0 \leq & \left\langle u_{n}-u_{n-1}, r_{n}\left(D x_{n-1}-D x_{n}\right)+\frac{r_{n}}{r_{n-1}}\left(u_{n-1}-x_{n-1}\right)-\left(u_{n}-x_{n}\right)\right\rangle \\
= & \left\langle u_{n-1}-u_{n}, u_{n}-u_{n-1}+\left(1-\frac{r_{n}}{r_{n-1}}\right) u_{n-1}\right. \\
& \left.+\left(x_{n-1}-r_{n} D x_{n-1}\right)-\left(x_{n}-r_{n} D x_{n}\right)-x_{n-1}+\frac{r_{n}}{r_{n-1}} x_{n-1}\right\rangle \\
= & \left\langle u_{n-1}-u_{n},\left(1-\frac{r_{n}}{r_{n-1}}\right) u_{n-1}+\left(x_{n-1}-r_{n} D x_{n-1}\right)-\left(x_{n}-r_{n} D x_{n}\right)-x_{n-1}+\frac{r_{n}}{r_{n-1}} x_{n-1}\right\rangle \\
& -\left\|u_{n}-u_{n-1}\right\|^{2} \\
= & \left\langle u_{n-1}-u_{n},\left(1-\frac{r_{n}}{r_{n-1}}\right)\left(u_{n-1}-x_{n-1}\right)+\left(x_{n-1}-r_{n} D x_{n-1}\right)-\left(x_{n}-r_{n} D x_{n}\right)\right\rangle \\
& -\left\|u_{n}-u_{n-1}\right\|^{2} \\
\leq & \left\|u_{n-1}-u_{n}\right\|\left\{\left|1-\frac{r_{n}}{r_{n-1}}\right|\left\|u_{n-1}-x_{n-1}\right\|+\left\|\left(x_{n-1}-r_{n} D x_{n-1}\right)-\left(x_{n}-r_{n} D x_{n}\right)\right\|\right\} \\
& -\left\|u_{n}-u_{n-1}\right\|^{2} \\
\leq & \left\|u_{n-1}-u_{n}\right\|\left\{\left|1-\frac{r_{n}}{r_{n-1}}\right|\left\|u_{n-1}-x_{n-1}\right\|+\left\|x_{n-1}-x_{n}\right\|\right\}-\left\|u_{n}-u_{n-1}\right\|^{2},
\end{aligned}
$$


and then

$$
\left\|u_{n-1}-u_{n}\right\| \leq\left|1-\frac{r_{n}}{r_{n-1}}\right|\left\|u_{n-1}-x_{n-1}\right\|+\left\|x_{n-1}-x_{n}\right\| .
$$

Without loss of generality, let us assume that there exists a real number $\mu$ such that $r_{n}>$ $\mu>0$ for all positive integers $n$. Then we get

$$
\left\|u_{n-1}-u_{n}\right\| \leq\left\|x_{n-1}-x_{n}\right\|+\frac{1}{\mu}\left|r_{n-1}-r_{n}\right|\left\|u_{n-1}-x_{n-1}\right\| .
$$

It follows from (3.5) and (3.10) that

$$
\begin{aligned}
\left\|y_{n}-y_{n-1}\right\| \leq & \beta_{n}\left\|x_{n}-x_{n-1}\right\|+\left(1-\beta_{n}\right)\left\{\left\|x_{n}-x_{n-1}\right\|+\frac{1}{\mu}\left|r_{n}-r_{n-1}\right|\left\|u_{n-1}-x_{n-1}\right\|\right. \\
& \left.+\left|\lambda_{n}-\lambda_{n-1}\right|\left\|A u_{n-1}\right\|\right\}+\left|\beta_{n}-\beta_{n-1}\right|\left(\left\|S x_{n-1}\right\|+\left\|z_{n-1}\right\|\right) \\
= & \left\|x_{n}-x_{n-1}\right\|+\left(1-\beta_{n}\right)\left\{\frac{1}{\mu}\left|r_{n}-r_{n-1}\right|\left\|u_{n-1}-x_{n-1}\right\|+\left|\lambda_{n}-\lambda_{n-1}\right|\left\|A u_{n-1}\right\|\right\} \\
& +\left|\beta_{n}-\beta_{n-1}\right|\left(\left\|S x_{n-1}\right\|+\left\|z_{n-1}\right\|\right) .
\end{aligned}
$$

Next, we estimate

$$
\begin{aligned}
\left\|x_{n+1}-x_{n}\right\|= & \left\|P_{C}\left[V_{n}\right]-P_{C}\left[V_{n-1}\right]\right\| \\
\leq & \| \alpha_{n} \rho\left(U\left(x_{n}\right)-U\left(x_{n-1}\right)\right)+\left(\alpha_{n}-\alpha_{n-1}\right) \rho U\left(x_{n-1}\right) \\
& +\gamma_{n}\left(x_{n}-x_{n-1}\right)+\left(\gamma_{n}-\gamma_{n-1}\right) x_{n-1} \\
& +\left(1-\gamma_{n}\right)\left[\left(I-\frac{\alpha_{n} \mu}{1-\gamma_{n}} F\right)\left(T\left(y_{n}\right)\right)-\left(I-\frac{\alpha_{n} \mu}{1-\gamma_{n}} F\right) T\left(y_{n-1}\right)\right] \\
& +\left(\left(1-\gamma_{n}\right) I-\alpha_{n} \mu F\right)\left(T\left(y_{n-1}\right)\right)-\left(\left(1-\gamma_{n-1}\right) I-\alpha_{n-1} \mu F\right)\left(T\left(y_{n-1}\right)\right) \| \\
\leq & \alpha_{n} \rho \tau\left\|x_{n}-x_{n-1}\right\|+\gamma_{n}\left\|x_{n}-x_{n-1}\right\|+\left(1-\gamma_{n}\right)\left(1-\frac{\alpha_{n} \nu}{1-\gamma_{n}}\right)\left\|y_{n}-y_{n-1}\right\| \\
& +\left|\gamma_{n}-\gamma_{n-1}\right|\left(\left\|x_{n-1}\right\|+\left\|T\left(y_{n-1}\right)\right\|\right) \\
& +\left|\alpha_{n}-\alpha_{n-1}\right|\left(\left\|\rho U\left(x_{n-1}\right)\right\|+\left\|\mu F\left(T\left(y_{n-1}\right)\right)\right\|\right),
\end{aligned}
$$

where the second inequality follows from Lemma 2.5. From (3.11) and (3.12), we have

$$
\begin{aligned}
\left\|x_{n+1}-x_{n}\right\| \leq & \alpha_{n} \rho \tau\left\|x_{n}-x_{n-1}\right\|+\gamma_{n}\left\|x_{n}-x_{n-1}\right\| \\
& +\left(1-\gamma_{n}-\alpha_{n} v\right)\left\{\left\|x_{n}-x_{n-1}\right\|+\frac{1}{\mu}\left|r_{n}-r_{n-1}\right|\left\|u_{n-1}-x_{n-1}\right\|\right. \\
& \left.+\left|\lambda_{n}-\lambda_{n-1}\right|\left\|A u_{n-1}\right\|\right\} \\
& +\left|\beta_{n}-\beta_{n-1}\right|\left(\left\|S x_{n-1}\right\|+\left\|z_{n-1}\right\|\right)+\left|\gamma_{n}-\gamma_{n-1}\right|\left(\left\|x_{n-1}\right\|+\left\|T\left(y_{n-1}\right)\right\|\right) \\
& +\left|\alpha_{n}-\alpha_{n-1}\right|\left(\left\|\rho U\left(x_{n-1}\right)\right\|+\left\|\mu F\left(T\left(y_{n-1}\right)\right)\right\|\right)
\end{aligned}
$$




$$
\begin{aligned}
\leq & \left(1-(v-\rho \tau) \alpha_{n}\right)\left\|x_{n}-x_{n-1}\right\|+\frac{1}{\mu}\left|r_{n}-r_{n-1}\right|\left\|u_{n-1}-x_{n-1}\right\| \\
& +\left|\lambda_{n}-\lambda_{n-1}\right|\left\|A u_{n-1}\right\| \\
& +\left|\beta_{n}-\beta_{n-1}\right|\left(\left\|S x_{n-1}\right\|+\left\|z_{n-1}\right\|\right)+\left|\gamma_{n}-\gamma_{n-1}\right|\left(\left\|x_{n-1}\right\|+\left\|T\left(y_{n-1}\right)\right\|\right) \\
& +\left|\alpha_{n}-\alpha_{n-1}\right|\left(\left\|\rho U\left(x_{n-1}\right)\right\|+\left\|\mu F\left(T\left(y_{n-1}\right)\right)\right\|\right) \\
\leq & \left(1-(v-\rho \tau) \alpha_{n}\right)\left\|x_{n}-x_{n-1}\right\| \\
& +M\left(\frac{1}{\mu}\left|r_{n-1}-r_{n}\right|+\left|\lambda_{n}-\lambda_{n-1}\right|+\left|\beta_{n}-\beta_{n-1}\right|+\left|\gamma_{n}-\gamma_{n-1}\right|\right. \\
& \left.+\left|\alpha_{n}-\alpha_{n-1}\right|\right) .
\end{aligned}
$$

Here

$$
\begin{aligned}
M= & \max \left\{\sup _{n \geq 1}\left\|u_{n-1}-x_{n-1}\right\|, \sup _{n \geq 1}\left\|A u_{n-1}\right\|, \sup _{n \geq 1}\left(\left\|S x_{n-1}\right\|+\left\|z_{n-1}\right\|\right),\right. \\
& \left.\sup _{n \geq 1}\left(\left\|x_{n-1}\right\|+\left\|T\left(y_{n-1}\right)\right\|\right), \sup _{n \geq 1}\left(\left\|\rho U\left(x_{n-1}\right)\right\|+\left\|\mu F\left(T\left(y_{n-1}\right)\right)\right\|\right)\right\} .
\end{aligned}
$$

It follows by conditions (a)-(e) of Algorithm 3.1 and Lemma 2.6 that

$$
\lim _{n \rightarrow \infty}\left\|x_{n+1}-x_{n}\right\|=0
$$

Next, we show that $\lim _{n \rightarrow \infty}\left\|u_{n}-x_{n}\right\|=0$. Since $x^{*} \in F(T) \cap V I(C, D) \cap \operatorname{GMEP}(F, \varphi, D)$, by using (3.1) and (3.2), we obtain

$$
\begin{aligned}
\left\|x_{n+1}-x^{*}\right\|^{2}= & \left\langle P_{C}\left[V_{n}\right]-x^{*}, x_{n+1}-x^{*}\right\rangle \\
= & \left\langle P_{C}\left[V_{n}\right]-V_{n}, P_{C}\left[V_{n}\right]-x^{*}\right\rangle+\left\langle V_{n}-x^{*}, x_{n+1}-x^{*}\right\rangle \\
\leq & \left\langle\alpha_{n}\left(\rho U\left(x_{n}\right)-\mu F\left(x^{*}\right)\right)+\gamma_{n}\left(x_{n}-x^{*}\right)+\left(\left(1-\gamma_{n}\right) I-\alpha_{n} \mu F\right)\left(T\left(y_{n}\right)\right)\right. \\
& \left.-\left(\left(1-\gamma_{n}\right) I-\alpha_{n} \mu F\right)\left(T\left(x^{*}\right)\right), x_{n+1}-x^{*}\right\rangle \\
= & \left\langle\alpha_{n} \rho\left(U\left(x_{n}\right)-U\left(x^{*}\right)\right), x_{n+1}-x^{*}\right\rangle+\alpha_{n}\left\langle\rho U\left(x^{*}\right)-\mu F\left(x^{*}\right), x_{n+1}-x^{*}\right\rangle \\
& +\left\langle\gamma_{n}\left(x_{n}-x^{*}\right), x_{n+1}-x^{*}\right\rangle \\
& +\left(1-\gamma_{n}\right)\left\langle\left(I-\frac{\alpha_{n} \mu}{1-\gamma_{n}} F\right)\left(T\left(y_{n}\right)\right)-\left(I-\frac{\alpha_{n} \mu}{1-\gamma_{n}} F\right)\left(T\left(x^{*}\right)\right), x_{n+1}-x^{*}\right\rangle \\
\leq & \left(\alpha_{n} \rho \tau+\gamma_{n}\right)\left\|x_{n}-x^{*}\right\|\left\|x_{n+1}-x^{*}\right\|+\alpha_{n}\left\langle\rho U\left(x^{*}\right)-\mu F\left(x^{*}\right), x_{n+1}-x^{*}\right\rangle \\
& +\left(1-\gamma_{n}-\alpha_{n} v\right)\left\|y_{n}-x^{*}\right\|\left\|x_{n+1}-x^{*}\right\| \\
\leq & \frac{\gamma_{n}+\alpha_{n} \rho \tau}{2}\left(\left\|x_{n}-x^{*}\right\|^{2}+\left\|x_{n+1}-x^{*}\right\|^{2}\right) \\
& +\alpha_{n}\left\langle\rho U\left(x^{*}\right)-\mu F\left(x^{*}\right), x_{n+1}-x^{*}\right\rangle \\
& +\frac{\left(1-\gamma_{n}-\alpha_{n} v\right)}{2}\left(\left\|y_{n}-x^{*}\right\|^{2}+\left\|x_{n+1}-x^{*}\right\|^{2}\right) \\
\leq & \frac{\left(1-\alpha_{n}(v-\rho \tau)\right)}{2}\left\|x_{n+1}-x^{*}\right\|^{2}+\frac{\gamma_{n}+\alpha_{n} \rho \tau}{2}\left\|x_{n}-x^{*}\right\|^{2} \\
& +\alpha_{n}\left\langle\rho U\left(x^{*}\right)-\mu F\left(x^{*}\right), x_{n+1}-x^{*}\right\rangle
\end{aligned}
$$




$$
\begin{aligned}
& +\frac{\left(1-\gamma_{n}-\alpha_{n} v\right)}{2}\left(\beta_{n}\left\|S x_{n}-x^{*}\right\|^{2}+\left(1-\beta_{n}\right)\left\|z_{n}-x^{*}\right\|^{2}\right) \\
\leq & \frac{\left(1-\alpha_{n}(\nu-\rho \tau)\right)}{2}\left\|x_{n+1}-x^{*}\right\|^{2}+\frac{\gamma_{n}+\alpha_{n} \rho \tau}{2}\left\|x_{n}-x^{*}\right\|^{2} \\
& +\alpha_{n}\left\langle\rho U\left(x^{*}\right)-\mu F\left(x^{*}\right), x_{n+1}-x^{*}\right\rangle+\frac{\left(1-\gamma_{n}-\alpha_{n} v\right) \beta_{n}}{2}\left\|S x_{n}-x^{*}\right\|^{2} \\
& +\frac{\left(1-\gamma_{n}-\alpha_{n} v\right)\left(1-\beta_{n}\right)}{2}\left\{\left\|u_{n}-x^{*}\right\|^{2}-\lambda_{n}\left(2 \alpha-\lambda_{n}\right)\left\|A u_{n}-A x^{*}\right\|^{2}\right\} \\
\leq & \frac{\left(1-\alpha_{n}(\nu-\rho \tau)\right)}{2}\left\|x_{n+1}-x^{*}\right\|^{2}+\frac{\gamma_{n}+\alpha_{n} \rho \tau}{2}\left\|x_{n}-x^{*}\right\|^{2} \\
& +\alpha_{n}\left(\rho U\left(x^{*}\right)-\mu F\left(x^{*}\right), x_{n+1}-x^{*}\right\rangle+\frac{\left(1-\gamma_{n}-\alpha_{n} v\right) \beta_{n}}{2}\left\|S x_{n}-x^{*}\right\|^{2} \\
& +\frac{\left(1-\gamma_{n}-\alpha_{n} \nu\right)\left(1-\beta_{n}\right)}{2}\left\{\left\|x_{n}-x^{*}\right\|^{2}-r_{n}\left(2 \theta-r_{n}\right)\left\|D x_{n}-D x^{*}\right\|^{2}\right. \\
& \left.-\lambda_{n}\left(2 \alpha-\lambda_{n}\right)\left\|A u_{n}-A x^{*}\right\|^{2}\right\},
\end{aligned}
$$

which implies that

$$
\begin{aligned}
\left\|x_{n+1}-x^{*}\right\|^{2} \leq & \frac{\gamma_{n}+\alpha_{n} \rho \tau}{1+\alpha_{n}(v-\rho \tau)}\left\|x_{n}-x^{*}\right\|^{2} \\
& +\frac{2 \alpha_{n}}{1+\alpha_{n}(v-\rho \tau)}\left\langle\rho U\left(x^{*}\right)-\mu F\left(x^{*}\right), x_{n+1}-x^{*}\right\rangle \\
& +\frac{\left(1-\gamma_{n}-\alpha_{n} v\right) \beta_{n}}{1+\alpha_{n}(v-\rho \tau)}\left\|S x_{n}-x^{*}\right\|^{2} \\
& +\frac{\left(1-\gamma_{n}-\alpha_{n} v\right)\left(1-\beta_{n}\right)}{1+\alpha_{n}(v-\rho \tau)}\left\{\left\|x_{n}-x^{*}\right\|^{2}-r_{n}\left(2 \theta-r_{n}\right)\left\|D x_{n}-D x^{*}\right\|^{2}\right. \\
& \left.-\lambda_{n}\left(2 \alpha-\lambda_{n}\right)\left\|A u_{n}-A x^{*}\right\|^{2}\right\} \\
\leq & \frac{\gamma_{n}+\alpha_{n} \rho \tau}{1+\alpha_{n}(v-\rho \tau)}\left\|x_{n}-x^{*}\right\|^{2} \\
& +\frac{2 \alpha_{n}}{1+\alpha_{n}(v-\rho \tau)}\left\langle\rho U\left(x^{*}\right)-\mu F\left(x^{*}\right), x_{n+1}-x^{*}\right\rangle \\
& +\left\|x_{n}-x^{*}\right\|^{2}+\frac{\left(1-\gamma_{n}-\alpha_{n} v\right) \beta_{n}}{1+\alpha_{n}(\nu-\rho \tau)}\left\|S x_{n}-x^{*}\right\|^{2} \\
& -\frac{\left(1-\gamma_{n}-\alpha_{n} v\right)\left(1-\beta_{n}\right)}{1+\alpha_{n}(v-\rho \tau)}\left\{r_{n}\left(2 \theta-r_{n}\right)\left\|D x_{n}-D x^{*}\right\|^{2}\right. \\
& \left.+\lambda_{n}\left(2 \alpha-\lambda_{n}\right)\left\|A u_{n}-A x^{*}\right\|^{2}\right\} .
\end{aligned}
$$

Then from the above inequality, we get

$$
\begin{aligned}
& \frac{\left(1-\gamma_{n}-\alpha_{n} \nu\right)\left(1-\beta_{n}\right)}{1+\alpha_{n}(v-\rho \tau)}\left\{r_{n}\left(2 \theta-r_{n}\right)\left\|D x_{n}-D x^{*}\right\|^{2}\right. \\
& \left.\quad+\lambda_{n}\left(2 \alpha-\lambda_{n}\right)\left\|A u_{n}-A x^{*}\right\|^{2}\right\} \\
& \leq \frac{\gamma_{n}+\alpha_{n} \rho \tau}{1+\alpha_{n}(v-\rho \tau)}\left\|x_{n}-x^{*}\right\|^{2}+\frac{2 \alpha_{n}}{1+\alpha_{n}(\nu-\rho \tau)}\left\langle\rho U\left(x^{*}\right)-\mu F\left(x^{*}\right), x_{n+1}-x^{*}\right\rangle \\
& \quad+\beta_{n}\left\|S x_{n}-x^{*}\right\|^{2}+\left\|x_{n}-x^{*}\right\|^{2}-\left\|x_{n+1}-x^{*}\right\|^{2}
\end{aligned}
$$




$$
\begin{aligned}
\leq & \frac{\gamma_{n}+\alpha_{n} \rho \tau}{1+\alpha_{n}(v-\rho \tau)}\left\|x_{n}-x^{*}\right\|^{2}+\frac{2 \alpha_{n}}{1+\alpha_{n}(v-\rho \tau)}\left\langle\rho U\left(x^{*}\right)-\mu F\left(x^{*}\right), x_{n+1}-x^{*}\right\rangle \\
& +\beta_{n}\left\|S x_{n}-x^{*}\right\|^{2}+\left(\left\|x_{n}-x^{*}\right\|+\left\|x_{n+1}-x^{*}\right\|\right)\left\|x_{n+1}-x_{n}\right\| .
\end{aligned}
$$

Since $\liminf _{n \rightarrow \infty} \lambda_{n} \leq \limsup _{n \rightarrow \infty} \lambda_{n}<2 \alpha,\left\{r_{n}\right\} \subset(0,2 \theta), \lim _{n \rightarrow \infty}\left\|x_{n+1}-x_{n}\right\|=0, \gamma_{n} \rightarrow 0$, $\alpha_{n} \rightarrow 0$, and $\beta_{n} \rightarrow 0$, we obtain $\lim _{n \rightarrow \infty}\left\|D x_{n}-D x^{*}\right\|=0$ and $\lim _{n \rightarrow \infty}\left\|A u_{n}-A x^{*}\right\|=0$.

Since $T_{r_{n}}$ is firmly nonexpansive, we have

$$
\begin{aligned}
\left\|u_{n}-x^{*}\right\|^{2}= & \left\|T_{r_{n}}\left(x_{n}-r_{n} D x_{n}\right)-T_{r_{n}}\left(x^{*}-r_{n} D x^{*}\right)\right\|^{2} \\
\leq & \left\langle u_{n}-x^{*},\left(x_{n}-r_{n} D x_{n}\right)-\left(x^{*}-r_{n} D x^{*}\right)\right\rangle \\
= & \frac{1}{2}\left\{\left\|u_{n}-x^{*}\right\|^{2}+\left\|\left(x_{n}-r_{n} D x_{n}\right)-\left(x^{*}-r_{n} D x^{*}\right)\right\|^{2}\right. \\
& \left.-\left\|u_{n}-x^{*}-\left[\left(x_{n}-r_{n} D x_{n}\right)-\left(x^{*}-r_{n} D x^{*}\right)\right]\right\|^{2}\right\} .
\end{aligned}
$$

Hence, we get

$$
\begin{aligned}
\left\|u_{n}-x^{*}\right\|^{2} & \leq\left\|\left(x_{n}-r_{n} D x_{n}\right)-\left(x^{*}-r_{n} D x^{*}\right)\right\|^{2}-\left\|u_{n}-x_{n}+r_{n}\left(D x_{n}-D x^{*}\right)\right\|^{2} \\
& \leq\left\|x_{n}-x^{*}\right\|^{2}-\left\|u_{n}-x_{n}+r_{n}\left(D x_{n}-D x^{*}\right)\right\|^{2} \\
& \leq\left\|x_{n}-x^{*}\right\|^{2}-\left\|u_{n}-x_{n}\right\|^{2}+2 r_{n}\left\|u_{n}-x_{n}\right\|\left\|D x_{n}-D x^{*}\right\| .
\end{aligned}
$$

From (3.14), (3.2), and the above inequality, we have

$$
\begin{aligned}
\left\|x_{n+1}-x^{*}\right\|^{2} \leq & \frac{\left(1-\alpha_{n}(v-\rho \tau)\right)}{2}\left\|x_{n+1}-x^{*}\right\|^{2}+\frac{\gamma_{n}+\alpha_{n} \rho \tau}{2}\left\|x_{n}-x^{*}\right\|^{2} \\
& +\alpha_{n}\left(\rho U\left(x^{*}\right)-\mu F\left(x^{*}\right), x_{n+1}-x^{*}\right\rangle \\
& +\frac{\left(1-\gamma_{n}-\alpha_{n} v\right)}{2}\left(\beta_{n}\left\|S x_{n}-x^{*}\right\|^{2}+\left(1-\beta_{n}\right)\left\|z_{n}-x^{*}\right\|^{2}\right) \\
\leq & \frac{\left(1-\alpha_{n}(\nu-\rho \tau)\right)}{2}\left\|x_{n+1}-x^{*}\right\|^{2}+\frac{\gamma_{n}+\alpha_{n} \rho \tau}{2}\left\|x_{n}-x^{*}\right\|^{2} \\
& +\alpha_{n}\left(\rho U\left(x^{*}\right)-\mu F\left(x^{*}\right), x_{n+1}-x^{*}\right\rangle \\
& +\frac{\left(1-\gamma_{n}-\alpha_{n} v\right)}{2}\left(\beta_{n}\left\|S x_{n}-x^{*}\right\|^{2}+\left(1-\beta_{n}\right)\left\|u_{n}-x^{*}\right\|^{2}\right) \\
\leq & \frac{\left(1-\alpha_{n}(v-\rho \tau)\right)}{2}\left\|x_{n+1}-x^{*}\right\|^{2}+\frac{\gamma_{n}+\alpha_{n} \rho \tau}{2}\left\|x_{n}-x^{*}\right\|^{2} \\
& +\alpha_{n}\left(\rho U\left(x^{*}\right)-\mu F\left(x^{*}\right), x_{n+1}-x^{*}\right\rangle \\
& +\frac{\left(1-\gamma_{n}-\alpha_{n} v\right)}{2}\left\{\beta_{n}\left\|S x_{n}-x^{*}\right\|^{2}+\left(1-\beta_{n}\right)\left(\left\|x_{n}-x^{*}\right\|^{2}-\left\|u_{n}-x_{n}\right\|^{2}\right.\right. \\
& \left.\left.+2 r_{n}\left\|u_{n}-x_{n}\right\|\left\|D x_{n}-D x^{*}\right\|\right)\right\},
\end{aligned}
$$

which implies that

$$
\begin{aligned}
\left\|x_{n+1}-x^{*}\right\|^{2} \leq & \frac{\gamma_{n}+\alpha_{n} \rho \tau}{1+\alpha_{n}(\nu-\rho \tau)}\left\|x_{n}-x^{*}\right\|^{2} \\
& +\frac{2 \alpha_{n}}{1+\alpha_{n}(v-\rho \tau)}\left\langle\rho U\left(x^{*}\right)-\mu F\left(x^{*}\right), x_{n+1}-x^{*}\right\rangle
\end{aligned}
$$




$$
\begin{aligned}
+ & \frac{\left(1-\gamma_{n}-\alpha_{n} v\right) \beta_{n}}{1+\alpha_{n}(\nu-\rho \tau)}\left\|S x_{n}-x^{*}\right\|^{2} \\
& +\frac{\left(1-\gamma_{n}-\alpha_{n} v\right)\left(1-\beta_{n}\right)}{1+\alpha_{n}(v-\rho \tau)}\left\{\left\|x_{n}-x^{*}\right\|^{2}-\left\|u_{n}-x_{n}\right\|^{2}\right. \\
& \left.+2 r_{n}\left\|u_{n}-x_{n}\right\|\left\|D x_{n}-D x^{*}\right\|\right\} \\
\leq & \frac{\gamma_{n}+\alpha_{n} \rho \tau}{1+\alpha_{n}(v-\rho \tau)}\left\|x_{n}-x^{*}\right\|^{2} \\
& +\frac{2 \alpha_{n}}{1+\alpha_{n}(v-\rho \tau)}\left\{\rho U\left(x^{*}\right)-\mu F\left(x^{*}\right), x_{n+1}-x^{*}\right\rangle \\
& +\frac{\left(1-\gamma_{n}-\alpha_{n} v\right) \beta_{n}}{1+\alpha_{n}(v-\rho \tau)}\left\|S x_{n}-x^{*}\right\|^{2} \\
& +\left\|x_{n}-x^{*}\right\|^{2}+\frac{\left(1-\gamma_{n}-\alpha_{n} \nu\right)\left(1-\beta_{n}\right)}{1+\alpha_{n}(v-\rho \tau)} \\
& \times\left\{-\left\|u_{n}-x_{n}\right\|^{2}+2 r_{n}\left\|u_{n}-x_{n}\right\|\left\|D x_{n}-D x^{*}\right\|\right\} .
\end{aligned}
$$

Hence

$$
\begin{aligned}
& \frac{\left(1-\gamma_{n}-\alpha_{n} v\right)\left(1-\beta_{n}\right)}{1+\alpha_{n}(v-\rho \tau)}\left\|u_{n}-x_{n}\right\|^{2} \\
& \leq \frac{\gamma_{n}+\alpha_{n} \rho \tau}{1+\alpha_{n}(v-\rho \tau)}\left\|x_{n}-x^{*}\right\|^{2}+\frac{2 \alpha_{n}}{1+\alpha_{n}(v-\rho \tau)}\left\langle\rho U\left(x^{*}\right)-\mu F\left(x^{*}\right), x_{n+1}-x^{*}\right\rangle \\
& \quad+\frac{\left(1-\gamma_{n}-\alpha_{n} v\right) \beta_{n}}{1+\alpha_{n}(v-\rho \tau)}\left\|S x_{n}-x^{*}\right\|^{2} \\
& \quad+\frac{2\left(1-\gamma_{n}-\alpha_{n} v\right)\left(1-\beta_{n}\right) r_{n}}{1+\alpha_{n}(v-\rho \tau)}\left\|u_{n}-x_{n}\right\|\left\|D x_{n}-D x^{*}\right\|+\left\|x_{n}-x^{*}\right\|^{2}-\left\|x_{n+1}-x^{*}\right\|^{2} \\
& \leq \\
& \quad \frac{\gamma_{n}+\alpha_{n} \rho \tau}{1+\alpha_{n}(v-\rho \tau)}\left\|x_{n}-x^{*}\right\|^{2}+\frac{2 \alpha_{n}}{1+\alpha_{n}(v-\rho \tau)}\left\langle\rho U\left(x^{*}\right)-\mu F\left(x^{*}\right), x_{n+1}-x^{*}\right\rangle \\
& \quad+\frac{\left(1-\gamma_{n}-\alpha_{n} v\right) \beta_{n}}{1+\alpha_{n}(v-\rho \tau)}\left\|S x_{n}-x^{*}\right\|^{2} \\
& \quad+\frac{2\left(1-\gamma_{n}-\alpha_{n} v\right)\left(1-\beta_{n}\right) r_{n}}{1+\alpha_{n}(v-\rho \tau)}\left\|u_{n}-x_{n}\right\|\left\|D x_{n}-D x^{*}\right\| \\
& \quad+\left(\left\|x_{n}-x^{*}\right\|+\left\|x_{n+1}-x^{*}\right\|\right)\left\|x_{n+1}-x_{n}\right\| .
\end{aligned}
$$

Since $\lim _{n \rightarrow \infty}\left\|x_{n+1}-x_{n}\right\|=0, \alpha_{n} \rightarrow 0, \beta_{n} \rightarrow 0$, and $\lim _{n \rightarrow \infty}\left\|D x_{n}-D x^{*}\right\|=0$, we obtain

$$
\lim _{n \rightarrow \infty}\left\|u_{n}-x_{n}\right\|=0
$$

From (2.2), we get

$$
\begin{aligned}
\left\|z_{n}-x^{*}\right\|^{2}= & \left\|P_{C}\left[u_{n}-\lambda_{n} A u_{n}\right]-P_{C}\left[x^{*}-\lambda_{n} A x^{*}\right]\right\|^{2} \\
\leq & \left\{z_{n}-x^{*},\left(u_{n}-\lambda_{n} A u_{n}\right)-\left(x^{*}-\lambda_{n} A x^{*}\right)\right\rangle \\
= & \frac{1}{2}\left\{\left\|z_{n}-x^{*}\right\|^{2}+\left\|u_{n}-x^{*}-\lambda_{n}\left(A u_{n}-A x^{*}\right)\right\|^{2}\right. \\
& \left.-\left\|u_{n}-x^{*}-\lambda_{n}\left(A u_{n}-A x^{*}\right)-\left(z_{n}-x^{*}\right)\right\|^{2}\right\}
\end{aligned}
$$




$$
\begin{aligned}
& \leq \frac{1}{2}\left\{\left\|z_{n}-x^{*}\right\|^{2}+\left\|u_{n}-x^{*}\right\|^{2}-\left\|u_{n}-z_{n}-\lambda_{n}\left(A u_{n}-A x^{*}\right)\right\|^{2}\right\} \\
& \leq \frac{1}{2}\left\{\left\|z_{n}-x^{*}\right\|^{2}+\left\|u_{n}-x^{*}\right\|^{2}-\left\|u_{n}-z_{n}\right\|^{2}+2 \lambda_{n}\left\langle u_{n}-z_{n}, A u_{n}-A x^{*}\right\rangle\right\} \\
& \leq \frac{1}{2}\left\{\left\|z_{n}-x^{*}\right\|^{2}+\left\|u_{n}-x^{*}\right\|^{2}-\left\|u_{n}-z_{n}\right\|^{2}+2 \lambda_{n}\left\|u_{n}-z_{n}\right\|\left\|A u_{n}-A x^{*}\right\|\right\} .
\end{aligned}
$$

Hence

$$
\begin{aligned}
\left\|z_{n}-x^{*}\right\|^{2} & \leq\left\|u_{n}-x^{*}\right\|^{2}-\left\|u_{n}-z_{n}\right\|^{2}+2 \lambda_{n}\left\|u_{n}-z_{n}\right\|\left\|A u_{n}-A x^{*}\right\| \\
& \leq\left\|x_{n}-x^{*}\right\|^{2}-\left\|u_{n}-z_{n}\right\|^{2}+2 \lambda_{n}\left\|u_{n}-z_{n}\right\|\left\|A u_{n}-A x^{*}\right\|
\end{aligned}
$$

From (3.14) and the inequality above, we have

$$
\begin{aligned}
\left\|x_{n+1}-x^{*}\right\|^{2} \leq & \frac{\left(1-\alpha_{n}(v-\rho \tau)\right)}{2}\left\|x_{n+1}-x^{*}\right\|^{2}+\frac{\gamma_{n}+\alpha_{n} \rho \tau}{2}\left\|x_{n}-x^{*}\right\|^{2} \\
& +\alpha_{n}\left\langle\rho U\left(x^{*}\right)-\mu F\left(x^{*}\right), x_{n+1}-x^{*}\right\rangle \\
& +\frac{\left(1-\gamma_{n}-\alpha_{n} \nu\right)}{2}\left(\beta_{n}\left\|S x_{n}-x^{*}\right\|^{2}+\left(1-\beta_{n}\right)\left\|z_{n}-x^{*}\right\|^{2}\right) \\
\leq & \frac{\left(1-\alpha_{n}(v-\rho \tau)\right)}{2}\left\|x_{n+1}-x^{*}\right\|^{2}+\frac{\gamma_{n}+\alpha_{n} \rho \tau}{2}\left\|x_{n}-x^{*}\right\|^{2} \\
& +\alpha_{n}\left\langle\rho U\left(x^{*}\right)-\mu F\left(x^{*}\right), x_{n+1}-x^{*}\right\rangle+\frac{\left(1-\gamma_{n}-\alpha_{n} \nu\right)}{2}\left\{\beta_{n}\left\|S x_{n}-x^{*}\right\|^{2}\right. \\
& +\left(1-\beta_{n}\right)\left(\left\|x_{n}-x^{*}\right\|^{2}-\left\|u_{n}-z_{n}\right\|^{2}\right. \\
& \left.\left.+2 \lambda_{n}\left\|u_{n}-z_{n}\right\|\left\|A u_{n}-A x^{*}\right\|\right)\right\}
\end{aligned}
$$

which implies that

$$
\begin{aligned}
\left\|x_{n+1}-x^{*}\right\|^{2} \leq & \frac{\gamma_{n}+\alpha_{n} \rho \tau}{1+\alpha_{n}(v-\rho \tau)}\left\|x_{n}-x^{*}\right\|^{2} \\
& +\frac{2 \alpha_{n}}{1+\alpha_{n}(v-\rho \tau)}\left\langle\rho U\left(x^{*}\right)-\mu F\left(x^{*}\right), x_{n+1}-x^{*}\right\rangle \\
& +\frac{\left(1-\gamma_{n}-\alpha_{n} v\right) \beta_{n}}{1+\alpha_{n}(v-\rho \tau)}\left\|S x_{n}-x^{*}\right\|^{2} \\
& +\frac{\left(1-\gamma_{n}-\alpha_{n} v\right)\left(1-\beta_{n}\right)}{1+\alpha_{n}(v-\rho \tau)}\left\{\left\|x_{n}-x^{*}\right\|^{2}-\left\|u_{n}-z_{n}\right\|^{2}\right. \\
& \left.+2 \lambda_{n}\left\|u_{n}-z_{n}\right\|\left\|A u_{n}-A x^{*}\right\|\right\} .
\end{aligned}
$$

Hence

$$
\begin{aligned}
& \frac{\left(1-\gamma_{n}-\alpha_{n} \nu\right)\left(1-\beta_{n}\right)}{1+\alpha_{n}(v-\rho \tau)}\left\|u_{n}-x_{n}\right\|^{2} \\
& \leq \frac{\gamma_{n}+\alpha_{n} \rho \tau}{1+\alpha_{n}(v-\rho \tau)}\left\|x_{n}-x^{*}\right\|^{2} \\
& \quad+\frac{2 \alpha_{n}}{1+\alpha_{n}(v-\rho \tau)}\left\langle\rho U\left(x^{*}\right)-\mu F\left(x^{*}\right), x_{n+1}-x^{*}\right\rangle \\
& \quad+\frac{\left(1-\gamma_{n}-\alpha_{n} \nu\right) \beta_{n}}{1+\alpha_{n}(v-\rho \tau)}\left\|S x_{n}-x^{*}\right\|^{2}+\left\|x_{n}-x^{*}\right\|^{2}-\left\|x_{n+1}-x^{*}\right\|^{2}
\end{aligned}
$$




$$
\begin{aligned}
& +2 \lambda_{n}\left\|u_{n}-z_{n}\right\|\left\|A u_{n}-A x^{*}\right\| \\
= & \frac{\gamma_{n}+\alpha_{n} \rho \tau}{1+\alpha_{n}(v-\rho \tau)}\left\|x_{n}-x^{*}\right\|^{2} \\
& +\frac{2 \alpha_{n}}{1+\alpha_{n}(v-\rho \tau)}\left\langle\rho U\left(x^{*}\right)-\mu F\left(x^{*}\right), x_{n+1}-x^{*}\right\rangle \\
& +\frac{\left(1-\gamma_{n}-\alpha_{n} v\right) \beta_{n}}{1+\alpha_{n}(v-\rho \tau)}\left\|S x_{n}-x^{*}\right\|^{2} \\
& +\left(\left\|x_{n}-x^{*}\right\|+\left\|x_{n+1}-x^{*}\right\|\right)\left\|x_{n+1}-x_{n}\right\| \\
& +2 \lambda_{n}\left\|u_{n}-z_{n}\right\|\left\|A u_{n}-A x^{*}\right\| .
\end{aligned}
$$

Since $\lim _{n \rightarrow \infty}\left\|x_{n+1}-x_{n}\right\|=0, \gamma_{n} \rightarrow 0, \alpha_{n} \rightarrow 0, \beta_{n} \rightarrow 0$, and $\lim _{n \rightarrow \infty}\left\|A u_{n}-A x^{*}\right\|=0$, we get

$$
\lim _{n \rightarrow \infty}\left\|u_{n}-z_{n}\right\|=0
$$

It follows from (3.15) and (3.17) that

$$
\begin{aligned}
\lim _{n \rightarrow \infty}\left\|x_{n}-z_{n}\right\| & =0, \\
\left\|x_{n}-T\left(y_{n}\right)\right\| & \leq\left\|x_{n}-x_{n+1}\right\|+\left\|x_{n+1}-T\left(y_{n}\right)\right\| \\
& =\left\|x_{n}-x_{n+1}\right\|+\left\|P_{C}\left[V_{n}\right]-P_{C}\left[T\left(y_{n}\right)\right]\right\| \\
& \leq\left\|x_{n}-x_{n+1}\right\|+\left\|\alpha_{n}\left(\rho U\left(x_{n}\right)-\mu F\left(T\left(y_{n}\right)\right)\right)+\gamma_{n}\left(x_{n}-T\left(y_{n}\right)\right)\right\| \\
& \leq\left\|x_{n}-x_{n+1}\right\|+\alpha_{n}\left\|\rho U\left(x_{n}\right)-\mu F\left(T\left(y_{n}\right)\right)\right\|+\gamma_{n}\left\|x_{n}-T\left(y_{n}\right)\right\|,
\end{aligned}
$$

which implies that

$$
\left\|x_{n}-T\left(y_{n}\right)\right\| \leq \frac{1}{1-\gamma_{n}}\left\|x_{n}-x_{n+1}\right\|+\frac{\alpha_{n}}{1-\gamma_{n}}\left\|\rho U\left(x_{n}\right)-\mu F\left(T\left(y_{n}\right)\right)\right\| .
$$

Since $\lim _{n \rightarrow \infty}\left\|x_{n+1}-x_{n}\right\|=0, \alpha_{n} \rightarrow 0$, we obtain

$$
\lim _{n \rightarrow \infty}\left\|x_{n}-T\left(y_{n}\right)\right\|=0
$$

Since $T\left(x_{n}\right) \in C$, we have

$$
\begin{aligned}
\left\|x_{n}-T\left(x_{n}\right)\right\| \leq & \left\|x_{n}-x_{n+1}\right\|+\left\|x_{n+1}-T\left(x_{n}\right)\right\| \\
= & \left\|x_{n}-x_{n+1}\right\|+\left\|P_{C}\left[V_{n}\right]-P_{C}\left[T\left(x_{n}\right)\right]\right\| \\
\leq & \left\|x_{n}-x_{n+1}\right\|+\| \alpha_{n}\left(\rho U\left(x_{n}\right)-\mu F\left(T\left(y_{n}\right)\right)\right) \\
& \quad+\gamma_{n}\left(x_{n}-T\left(y_{n}\right)\right)+T\left(y_{n}\right)-T\left(x_{n}\right) \| \\
\leq & \left\|x_{n}-x_{n+1}\right\|+\alpha_{n}\left\|\rho U\left(x_{n}\right)-\mu F\left(T\left(y_{n}\right)\right)\right\|+\gamma_{n}\left\|x_{n}-T\left(y_{n}\right)\right\|+\left\|y_{n}-x_{n}\right\| \\
\leq & \left\|x_{n}-x_{n+1}\right\|+\alpha_{n}\left\|\rho U\left(x_{n}\right)-\mu F\left(T\left(y_{n}\right)\right)\right\|+\gamma_{n}\left\|x_{n}-T\left(y_{n}\right)\right\| \\
& \quad+\left\|\beta_{n} S x_{n}+\left(1-\beta_{n}\right) z_{n}-x_{n}\right\|
\end{aligned}
$$




$$
\begin{aligned}
\leq & \left\|x_{n}-x_{n+1}\right\|+\alpha_{n}\left\|\rho U\left(x_{n}\right)-\mu F\left(T\left(y_{n}\right)\right)\right\|+\gamma_{n}\left\|x_{n}-T\left(y_{n}\right)\right\| \\
& +\beta_{n}\left\|S x_{n}-x_{n}\right\|+\left(1-\beta_{n}\right)\left\|z_{n}-x_{n}\right\| .
\end{aligned}
$$

Since $\lim _{n \rightarrow \infty}\left\|x_{n+1}-x_{n}\right\|=0, \gamma_{n} \rightarrow 0, \alpha_{n} \rightarrow 0, \beta_{n} \rightarrow 0, \lim _{n \rightarrow \infty}\left\|x_{n}-T\left(y_{n}\right)\right\|=0$, $\left\|\rho U\left(x_{n}\right)-\mu F\left(T\left(y_{n}\right)\right)\right\|$ and $\left\|S x_{n}-x_{n}\right\|$ are bounded and $\lim _{n \rightarrow \infty}\left\|x_{n}-z_{n}\right\|=0$, we obtain

$$
\lim _{n \rightarrow \infty}\left\|x_{n}-T\left(x_{n}\right)\right\|=0
$$

Since $\left\{x_{n}\right\}$ is bounded, without loss of generality we can assume that $x_{n} \rightarrow x^{*} \in C$. It follows from Lemma 2.3 that $x^{*} \in F(T)$. Therefore $w_{w}\left(x_{n}\right) \subset F(T)$.

Theorem 3.1 The sequence $\left\{x_{n}\right\}$ generated by Algorithm 3.1 converges strongly to $z$, which is the unique solution of the variational inequality

$$
\langle\rho U(z)-\mu F(z), x-z\rangle \leq 0, \quad \forall x \in V I(C, A) \cap \operatorname{GMEP}(F, \varphi, D) \cap F(T) .
$$

Proof Since $\left\{x_{n}\right\}$ is bounded $x_{n} \rightarrow w$ and from Lemma 3.2, we have $w \in F(T)$. Next, we show that $w \in \operatorname{GMEP}(F, \varphi, D)$. Since $u_{n}=T_{r_{n}}\left(x_{n}-r_{n} D x_{n}\right)$, we have

$$
F_{1}\left(u_{n}, y\right)+\varphi(y)-\varphi\left(u_{n}\right)+\left\langle D x_{n}, y-u_{n}\right\rangle+\frac{1}{r_{n}}\left\langle y-u_{n}, u_{n}-x_{n}\right\rangle \geq 0, \quad \forall y \in C .
$$

It follows from the monotonicity of $F_{1}$ that

$$
\varphi(y)-\varphi\left(u_{n}\right)+\left\langle D x_{n}, y-u_{n}\right\rangle+\frac{1}{r_{n}}\left\langle y-u_{n}, u_{n}-x_{n}\right\rangle \geq F_{1}\left(y, u_{n}\right), \quad \forall y \in C
$$

and

$$
\varphi(y)-\varphi\left(u_{n_{k}}\right)+\left\langle D x_{n_{k}}, y-u_{n_{k}}\right\rangle+\left\langle y-u_{n_{k}}, \frac{u_{n_{k}}-x_{n_{k}}}{r_{n_{k}}}\right\rangle \geq F_{1}\left(y, u_{n_{k}}\right), \quad \forall y \in C .
$$

Since $\lim _{n \rightarrow \infty}\left\|u_{n}-x_{n}\right\|=0$ and $x_{n} \rightarrow w$, it is easy to observe that $u_{n_{k}} \rightarrow w$. For any $0<t \leq 1$ and $y \in C$, let $y_{t}=t y+(1-t) w$, and we have $y_{t} \in C$. Then from (3.20), we obtain

$$
\begin{aligned}
\left\langle D y_{t}, y_{t}-u_{n_{k}}\right\rangle \geq & \varphi\left(u_{n_{k}}\right)-\varphi\left(y_{t}\right)+\left\langle D y_{t}, y_{t}-u_{n_{k}}\right\rangle \\
& -\left\langle D x_{n_{k}}, y_{t}-u_{n_{k}}\right\rangle-\left\langle y_{t}-u_{n_{k}}, \frac{u_{n_{k}}-x_{n_{k}}}{r_{n_{k}}}\right\rangle+F_{1}\left(y_{t}, u_{n_{k}}\right) \\
= & \varphi\left(u_{n_{k}}\right)-\varphi\left(y_{t}\right)+\left\langle D y_{t}-D u_{n_{k}}, y_{t}-u_{n_{k}}\right\rangle+\left\langle D u_{n_{k}}-D x_{n_{k}}, y_{t}-u_{n_{k}}\right\rangle \\
& -\left\langle y_{t}-u_{n_{k}}, \frac{u_{n_{k}}-x_{n_{k}}}{r_{n_{k}}}\right\rangle+F_{1}\left(y_{t}, u_{n_{k}}\right) .
\end{aligned}
$$

Since $D$ is Lipschitz continuous and $\lim _{n \rightarrow \infty}\left\|u_{n}-x_{n}\right\|=0$, we obtain $\lim _{k \rightarrow \infty} \| D u_{n_{k}}-$ $D x_{n_{k}} \|=0$. From the monotonicity of $D$, the weakly lower semicontinuity of $\varphi$ and $u_{n_{k}} \rightarrow w$, it follows from (3.21) that

$$
\left\langle D y_{t}, y_{t}-w\right\rangle \geq \varphi(w)-\varphi\left(y_{t}\right)+F_{1}\left(y_{t}, w\right) .
$$


Hence, from assumptions $\left(\mathrm{A}_{1}\right)-\left(\mathrm{A}_{4}\right)$ and (3.22), we have

$$
\begin{aligned}
0 & =F_{1}\left(y_{t}, y_{t}\right)+\varphi\left(y_{t}\right)-\varphi\left(y_{t}\right) \leq t F_{1}\left(y_{t}, y\right)+(1-t) F_{1}\left(y_{t}, w\right)+t \varphi(y)+(1-t) \varphi(w)-\varphi\left(y_{t}\right) \\
& =t\left[F_{1}\left(y_{t}, y\right)+\varphi(y)-\varphi\left(y_{t}\right)\right]+(1-t)\left[F_{1}\left(y_{t}, w\right)+\varphi(w)-\varphi\left(y_{t}\right)\right] \\
& \leq t\left[F_{1}\left(y_{t}, y\right)+\varphi(y)-\varphi\left(y_{t}\right)\right]+(1-t) t\left\langle D y_{t}, y-w\right\rangle
\end{aligned}
$$

which implies that $F_{1}\left(y_{t}, y\right)+\varphi(y)-\varphi\left(y_{t}\right)+(1-t)\left\langle D y_{t}, y-w\right\rangle \geq 0$. Letting $t \rightarrow 0_{+}$, we have

$$
F_{1}\left(y_{t}, y\right)+\varphi(y)-\varphi(w)+\langle D w, y-w\rangle \geq 0, \quad \forall y \in C
$$

which implies that $w \in G M E P(F, \varphi, D)$.

Furthermore, we show that $w \in \Omega^{*}$. Let

$$
T v= \begin{cases}A v+N_{C} v, & \forall v \in C, \\ \emptyset, & \text { otherwise }\end{cases}
$$

where $N_{C} v:=\{w \in H:\langle w, v-u\rangle \geq 0, \forall u \in C\}$ is the normal cone to $C$ at $v \in C$. Then $T$ is maximal monotone and $0 \in T v$ if and only if $v \in \Omega^{*}$ (see [31]). Let $G(T)$ denote the graph of $T$, and let $(v, u) \in G(T)$; since $u-A v \in N_{C} v$ and $z_{n} \in C$, we have

$$
\left\langle v-z_{n}, u-A v\right\rangle \geq 0
$$

On the other hand, it follows from $z_{n}=P_{C}\left[u_{n}-\lambda_{n} A u_{n}\right]$ and $v \in C$ that

$$
\left\langle v-z_{n}, z_{n}-\left(u_{n}-\lambda_{n} A u_{n}\right)\right\rangle \geq 0
$$

and

$$
\left\langle v-z_{n}, \frac{z_{n}-u_{n}}{\lambda_{n}}+A u_{n}\right\rangle \geq 0 .
$$

Therefore, from (3.24) and the inverse strong monotonicity of $A$, we have

$$
\begin{aligned}
\left\langle v-z_{n_{k}}, u\right\rangle & \geq\left\langle v-z_{n_{k}}, A v\right\rangle \\
& \geq\left\langle v-z_{n_{k}}, A v\right\rangle-\left\langle v-z_{n_{k}}, \frac{z_{n_{k}}-u_{n_{k}}}{\lambda_{n_{k}}}+A u_{n_{k}}\right\rangle \\
& \geq\left\langle v-z_{n_{k}}, A v-A z_{n_{k}}\right\rangle+\left\langle v-z_{n_{k}}, A z_{n_{k}}-A u_{n_{k}}\right\rangle-\left\langle v-z_{n_{k}}, \frac{z_{n_{k}}-u_{n_{k}}}{\lambda_{n_{k}}}\right\rangle \\
& \geq\left\langle v-z_{n_{k}}, A z_{n_{k}}-A u_{n_{k}}\right\rangle-\left\langle v-z_{n_{k}}, \frac{z_{n_{k}}-u_{n_{k}}}{\lambda_{n_{k}}}\right\rangle .
\end{aligned}
$$

Since $\lim _{n \rightarrow \infty}\left\|u_{n}-z_{n}\right\|=0$ and $u_{n_{k}} \rightarrow w$, it is easy to observe that $z_{n_{k}} \rightarrow w$. Hence, we obtain $\langle v-w, u\rangle \geq 0$. Since $T$ is maximal monotone, we have $w \in T^{-1} 0$, and hence $w \in$ $V I(C, A)$. Thus we have

$$
w \in V I(C, A) \cap \operatorname{GMEP}(F, \varphi, D) \cap F(T) .
$$


Observe that the constants satisfy $0 \leq \rho \tau<\nu$ and

$$
\begin{aligned}
k \geq \eta & \Longleftrightarrow k^{2} \geq \eta^{2} \\
& \Longleftrightarrow 1-2 \mu \eta+\mu^{2} k^{2} \geq 1-2 \mu \eta+\mu^{2} \eta^{2} \\
& \Longleftrightarrow \sqrt{1-\mu\left(2 \eta-\mu k^{2}\right)} \geq 1-\mu \eta \\
& \Longleftrightarrow \mu \eta \geq 1-\sqrt{1-\mu\left(2 \eta-\mu k^{2}\right)} \\
& \Longleftrightarrow \mu \eta \geq v ;
\end{aligned}
$$

therefore, from Lemma 2.4 , the operator $\mu F-\rho U$ is $\mu \eta-\rho \tau$-strongly monotone, and we get the uniqueness of the solution of the variational inequality (3.19) and denote it by $z \in V I(C, A) \cap \operatorname{GMEP}(F, \varphi, D) \cap F(T)$.

Next, we claim that $\limsup _{n \rightarrow \infty}\left\langle\rho U(z)-\mu F(z), x_{n}-z\right\rangle \leq 0$. Since $\left\{x_{n}\right\}$ is bounded, there exists a subsequence $\left\{x_{n_{k}}\right\}$ of $\left\{x_{n}\right\}$ such that

$$
\begin{aligned}
\limsup _{n \rightarrow \infty}\left\langle\rho U(z)-\mu F(z), x_{n}-z\right\rangle & =\limsup _{k \rightarrow \infty}\left\langle\rho U(z)-\mu F(z), x_{n_{k}}-z\right\rangle \\
& =\langle\rho U(z)-\mu F(z), w-z\rangle \leq 0 .
\end{aligned}
$$

Next, we show that $x_{n} \rightarrow z$. We have

$$
\begin{aligned}
& \left\|x_{n+1}-z\right\|^{2}=\left\langle P_{C}\left[V_{n}\right]-z, x_{n+1}-z\right\rangle \\
& =\left\langle P_{C}\left[V_{n}\right]-V_{n}, P_{C}\left[V_{n}\right]-z\right\rangle+\left\langle V_{n}-z, x_{n+1}-z\right\rangle \\
& \leq\left\langle\alpha_{n}\left(\rho U\left(x_{n}\right)-\mu F(z)\right)+\gamma_{n}\left(x_{n}-z\right)\right. \\
& \left.+\left(1-\gamma_{n}\right)\left[\left(I-\frac{\alpha_{n} \mu}{1-\gamma_{n}} F\right)\left(T\left(y_{n}\right)\right)-\left(I-\frac{\alpha_{n} \mu}{1-\gamma_{n}} F\right)(T(z))\right], x_{n+1}-z\right) \\
& =\left\langle\alpha_{n} \rho\left(U\left(x_{n}\right)-U(z)\right), x_{n+1}-z\right\rangle+\alpha_{n}\left\langle\rho U(z)-\mu F(z), x_{n+1}-z\right\rangle \\
& +\gamma_{n}\left\langle x_{n}-z, x_{n+1}-z\right\rangle \\
& +\left(1-\gamma_{n}\right)\left\langle\left(I-\frac{\alpha_{n} \mu}{1-\gamma_{n}} F\right)\left(T\left(y_{n}\right)\right)-\left(I-\frac{\alpha_{n} \mu}{1-\gamma_{n}} F\right)(T(z)), x_{n+1}-z\right\rangle \\
& \leq\left(\gamma_{n}+\alpha_{n} \rho \tau\right)\left\|x_{n}-z\right\|\left\|x_{n+1}-z\right\|+\alpha_{n}\left\langle\rho U(z)-\mu F(z), x_{n+1}-z\right\rangle \\
& +\left(1-\gamma_{n}-\alpha_{n} v\right)\left\|y_{n}-z\right\|\left\|x_{n+1}-z\right\| \\
& \leq\left(\gamma_{n}+\alpha_{n} \rho \tau\right)\left\|x_{n}-z\right\|\left\|x_{n+1}-z\right\|+\alpha_{n}\left\langle\rho U(z)-\mu F(z), x_{n+1}-z\right\rangle \\
& +\left(1-\gamma_{n}-\alpha_{n} \nu\right)\left\{\beta_{n}\left\|S x_{n}-S z\right\|+\beta_{n}\|S z-z\|+\left(1-\beta_{n}\right)\left\|z_{n}-z\right\|\right\} \\
& \times\left\|x_{n+1}-z\right\| \\
& \leq\left(\gamma_{n}+\alpha_{n} \rho \tau\right)\left\|x_{n}-z\right\|\left\|x_{n+1}-z\right\|+\alpha_{n}\left\langle\rho U(z)-\mu F(z), x_{n+1}-z\right\rangle \\
& +\left(1-\gamma_{n}-\alpha_{n} \nu\right)\left\{\beta_{n}\left\|x_{n}-z\right\|+\beta_{n}\|S z-z\|+\left(1-\beta_{n}\right)\left\|x_{n}-z\right\|\right\}\left\|x_{n+1}-z\right\| \\
& =\left(1-\alpha_{n}(\nu-\rho \tau)\right)\left\|x_{n}-z\right\|\left\|x_{n+1}-z\right\|+\alpha_{n}\left\langle\rho U(z)-\mu F(z), x_{n+1}-z\right\rangle \\
& +\left(1-\gamma_{n}-\alpha_{n} \nu\right) \beta_{n}\|S z-z\|\left\|x_{n+1}-z\right\|
\end{aligned}
$$




$$
\begin{aligned}
\leq & \frac{1-\alpha_{n}(v-\rho \tau)}{2}\left(\left\|x_{n}-z\right\|^{2}+\left\|x_{n+1}-z\right\|^{2}\right)+\alpha_{n}\left\langle\rho U(z)-\mu F(z), x_{n+1}-z\right\rangle \\
& +\left(1-\gamma_{n}-\alpha_{n} v\right) \beta_{n}\|S z-z\|\left\|x_{n+1}-z\right\|,
\end{aligned}
$$

which implies that

$$
\begin{aligned}
\left\|x_{n+1}-z\right\|^{2} \leq & \frac{1-\alpha_{n}(v-\rho \tau)}{1+\alpha_{n}(v-\rho \tau)}\left\|x_{n}-z\right\|^{2}+\frac{2 \alpha_{n}}{1+\alpha_{n}(v-\rho \tau)}\left\langle\rho U(z)-\mu F(z), x_{n+1}-z\right\rangle \\
& +\frac{2\left(1-\gamma_{n}-\alpha_{n} v\right) \beta_{n}}{1+\alpha_{n}(v-\rho \tau)}\|S z-z\|\left\|x_{n+1}-z\right\| \\
\leq & \left(1-\alpha_{n}(v-\rho \tau)\right)\left\|x_{n}-z\right\|^{2}+\frac{2 \alpha_{n}(v-\rho \tau)}{1+\alpha_{n}(v-\rho \tau)} \\
& \times\left\{\frac{1}{v-\rho \tau}\left\langle\rho U(z)-\mu F(z), x_{n+1}-z\right\rangle\right. \\
& \left.+\frac{\left(1-\gamma_{n}-\alpha_{n} v\right) \beta_{n}}{\alpha_{n}(v-\rho \tau)}\|S z-z\|\left\|x_{n+1}-z\right\|\right\} .
\end{aligned}
$$

Let $v_{n}=\alpha_{n}(v-\rho \tau)$ and $\delta_{n}=\frac{2 \alpha_{n}(v-\rho \tau)}{1+\alpha_{n}(\nu-\rho \tau)}\left\{\frac{1}{\nu-\rho \tau}\left\langle\rho U(z)-\mu F(z), x_{n+1}-z\right\rangle+\frac{\left(1-\gamma_{n}-\alpha_{n} v\right) \beta_{n}}{\alpha_{n}(\nu-\rho \tau)} \| S z-\right.$ $\left.z\|\| x_{n+1}-z \|\right\}$.

We have

$$
\sum_{n=1}^{\infty} \alpha_{n}=\infty
$$

and

$$
\limsup _{n \rightarrow \infty}\left\{\frac{1}{v-\rho \tau}\left\langle\rho U(z)-\mu F(z), x_{n+1}-z\right\rangle+\frac{\left(1-\gamma_{n}-\alpha_{n} \nu\right) \beta_{n}}{\alpha_{n}(v-\rho \tau)}\|S z-z\|\left\|x_{n+1}-z\right\|\right\} \leq 0
$$

It follows that

$$
\sum_{n=1}^{\infty} v_{n}=\infty \quad \text { and } \quad \limsup _{n \rightarrow \infty} \frac{\delta_{n}}{v_{n}} \leq 0
$$

Thus all the conditions of Lemma 2.6 are satisfied. Hence we deduce that $x_{n} \rightarrow z$. This completes the proof.

\section{Applications}

In this section, we obtain the following results by using a special case of the proposed method for example.

Putting $\gamma_{n}=0$ and $A=0$ in Algorithm 3.1, we obtain the following result which can be viewed as an extension and improvement of the method of Wang and $\mathrm{Xu}$ [30] for finding the approximate element of the common set of solutions of a generalized mixed equilibrium problem and a hierarchical fixed point problem in a real Hilbert space.

Corollary 4.1 Let $C$ be a nonempty closed convex subset of a real Hilbert space $H$. Let $D: C \rightarrow H$ be a $\theta$-inverse strongly monotone mapping. Let $F_{1}: C \times C \rightarrow \mathbb{R}$ be a bifunction satisfying assumptions (i)-(iv) of Lemma 2.2 and $S, T: C \rightarrow C$ be nonexpansive mappings 
such that $F(T) \cap \operatorname{MEP}\left(F_{1}\right) \neq \emptyset$. Let $F: C \rightarrow C$ be a $k$-Lipschitzian mapping and $\eta$-strongly monotone, and let $U: C \rightarrow C$ be a $\tau$-Lipschitzian mapping. For an arbitrarily given $x_{0} \in C$, let the iterative sequences $\left\{u_{n}\right\},\left\{x_{n}\right\},\left\{y_{n}\right\}$, and $\left\{z_{n}\right\}$ be generated by

$$
\begin{aligned}
& F_{1}\left(u_{n}, y\right)+\left\langle D x_{n}, y-u_{n}\right\rangle+\varphi(y)-\varphi\left(u_{n}\right)+\frac{1}{r_{n}}\left\langle y-u_{n}, u_{n}-x_{n}\right\rangle \geq 0, \quad \forall y \in C ; \\
& y_{n}=\beta_{n} S x_{n}+\left(1-\beta_{n}\right) u_{n} ; \\
& x_{n+1}=P_{C}\left[\alpha_{n} \rho U\left(x_{n}\right)+\left(I-\alpha_{n} \mu F\right)\left(T\left(y_{n}\right)\right)\right], \quad \forall n \geq 0,
\end{aligned}
$$

where $\left\{r_{n}\right\} \subset(0,2 \theta),\left\{\alpha_{n}\right\} \subset(0,1),\left\{\beta_{n}\right\} \subset(0,1)$. Suppose that the parameters satisfy $0<\mu<$ $\frac{2 \eta}{k^{2}}, 0 \leq \rho \tau<v$, where $v=1-\sqrt{1-\mu\left(2 \eta-\mu k^{2}\right)}$. Also $\left\{\alpha_{n}\right\},\left\{\beta_{n}\right\}$, and $\left\{r_{n}\right\}$ are sequences satisfying conditions (b)-(e) of Algorithm 3.1. The sequence $\left\{x_{n}\right\}$ converges strongly to $z$, which is the unique solution of the variational inequality

$$
\langle\rho U(z)-\mu F(z), x-z\rangle \leq 0, \quad \forall x \in M E P\left(F_{1}\right) \cap F(T) .
$$

Putting $U=f, F=I, \rho=\mu=1, \gamma_{n}=0$, and $A=0$, we obtain an extension and improvement of the method of Yao et al. [15] for finding the approximate element of the common set of solutions of a generalized mixed equilibrium problem and a hierarchical fixed point problem in a real Hilbert space.

Corollary 4.2 Let $C$ be a nonempty closed convex subset of a real Hilbert space $H$. Let $D: C \rightarrow H$ be a $\theta$-inverse strongly monotone mapping. Let $F_{1}: C \times C \rightarrow \mathbb{R}$ be a bifunction satisfying assumptions (i)-(iv) of Lemma 2.2 and $S, T: C \rightarrow C$ be nonexpansive mappings such that $F(T) \cap \operatorname{MEP}\left(F_{1}\right) \neq \emptyset$. Let $f: C \rightarrow C$ be a $\tau$-Lipschitzian mapping. For an arbitrarily given $x_{0} \in C$, let the iterative sequences $\left\{u_{n}\right\},\left\{x_{n}\right\},\left\{y_{n}\right\}$, and $\left\{z_{n}\right\}$ be generated by

$$
\begin{aligned}
& F_{1}\left(u_{n}, y\right)+\left\langle D x_{n}, y-u_{n}\right\rangle+\varphi(y)-\varphi\left(u_{n}\right)+\frac{1}{r_{n}}\left\langle y-u_{n}, u_{n}-x_{n}\right\rangle \geq 0, \quad \forall y \in C ; \\
& y_{n}=\beta_{n} S x_{n}+\left(1-\beta_{n}\right) u_{n} ; \\
& x_{n+1}=P_{C}\left[\alpha_{n} f\left(x_{n}\right)+\left(1-\alpha_{n}\right) T\left(y_{n}\right)\right], \quad \forall n \geq 0,
\end{aligned}
$$

where $\left\{r_{n}\right\} \subset(0,2 \theta),\left\{\alpha_{n}\right\},\left\{\beta_{n}\right\}$ are sequences in $(0,1)$ satisfying conditions (b)-(e) of Algorithm 3.1. The sequence $\left\{x_{n}\right\}$ converges strongly to $z$, which is the unique solution of the variational inequality

$$
\langle f(z)-z, x-z\rangle \leq 0, \quad \forall x \in M E P\left(F_{1}\right) \cap F(T) .
$$

Next, the following example shows that conditions (a)-(f) of Algorithm 3.1 are satisfied.

Example 4.1 Let $\alpha_{n}=\frac{1}{2} n^{-t}, \gamma_{n}=\frac{1}{2} n^{-t}, \beta_{n}=n^{-s}$ (with $\left.0<t<s \leq 1\right), \lambda_{n}=\frac{1}{2(n+1)}$, and $r_{n}=\frac{n}{n+1}$.

We have

$$
\begin{aligned}
& \alpha_{n}+\gamma_{n}=\frac{1}{n^{t}}<1, \\
& \lim _{n \rightarrow \infty} \alpha_{n}=\lim _{n \rightarrow \infty} \gamma_{n}=\lim _{n \rightarrow \infty} \frac{1}{2 n^{t}}=0
\end{aligned}
$$


and

$$
\sum_{n=1}^{\infty} \alpha_{n}=\sum_{n=1}^{\infty} \frac{1}{2 n^{t}}=\infty
$$

Conditions (a) and (b) are satisfied.

$$
\lim _{n \rightarrow \infty} \frac{\beta_{n}}{\alpha_{n}}=\lim _{n \rightarrow \infty} \frac{1}{2 n^{s-t}}=0
$$

Condition (c) is satisfied. We compute

$$
\alpha_{n-1}-\alpha_{n}=\frac{1}{2}\left(\frac{1}{(n-1)^{t}}-\frac{1}{n^{t}}\right)=\frac{1}{2(n-1)^{t}}\left(1-\left(1-\frac{1}{n}\right)^{t}\right) \sim \frac{t}{2 n^{t+1}} .
$$

It is easy to show $\sum_{n=1}^{\infty}\left|\alpha_{n-1}-\alpha_{n}\right|<\infty$. Similarly, we can show $\sum_{n=1}^{\infty}\left|\beta_{n-1}-\beta_{n}\right|<\infty$ and $\sum_{n=1}^{\infty}\left|\gamma_{n-1}-\gamma_{n}\right|<\infty$. The sequences $\left\{\alpha_{n}\right\},\left\{\gamma_{n}\right\}$, and $\left\{\beta_{n}\right\}$ satisfy condition (d). We have

$$
\liminf _{n \rightarrow \infty} r_{n}=\liminf _{n \rightarrow \infty} \frac{n}{n+1}=1
$$

and

$$
\begin{aligned}
\sum_{n=1}^{\infty}\left|r_{n-1}-r_{n}\right| & =\sum_{n=1}^{\infty}\left|\frac{n-1}{n}-\frac{n}{n+1}\right| \\
& =\sum_{n=1}^{\infty} \frac{1}{n(n+1)} \\
& \leq \sum_{n=1}^{\infty} \frac{1}{n^{2}} \\
& <\infty .
\end{aligned}
$$

Then the sequence $\left\{r_{n}\right\}$ satisfies condition (e). We compute

$$
\begin{aligned}
\sum_{n=1}^{\infty}\left|\lambda_{n-1}-\lambda_{n}\right|<\infty & =\sum_{n=1}^{\infty}\left|\frac{1}{2 n}-\frac{1}{2(n+1)}\right| \\
& =\frac{1}{2} .
\end{aligned}
$$

Then the sequence $\left\{\lambda_{n}\right\}$ satisfies condition (f).

Remark 4.1 In the hierarchical fixed point problem (1.9), if $S=I-(\rho U-\mu F)$, then we can get the variational inequality (3.19). In (3.19), if $U=0$ then we get the variational inequality $\langle F(z), x-z\rangle \geq 0, \forall x \in V I(C, A) \cap \operatorname{GMEP}(F, \varphi, D) \cap F(T)$, which just is the variational inequality studied by Suzuki [27] extending the common set of solutions of a system of variational inequalities, a generalized mixed equilibrium problem and a hierarchical fixed point problem. 


\section{Conclusions}

In this paper, we suggest and analyze an iterative method for finding the approximate element of the common set of solutions of (1.1), (1.5), and (1.9) in a real Hilbert space, which can be viewed as a refinement and improvement of some existing methods for solving a variational inequality problem, a generalized mixed equilibrium problem, and a hierarchical fixed point problem. Some existing methods (e.g., [15, 16, 18, 21, 23, 25]) can be viewed as special cases of Algorithm 3.1. Therefore, the new algorithm is expected to be widely applicable.

\section{Competing interests}

The authors declare that they have no competing interests.

\section{Authors' contributions}

All authors contributed equally and significantly in writing this article. All authors read and approved the final manuscript.

\section{Acknowledgements}

The authors are very grateful to the referees for their careful reading, comments, and suggestions, which improved the presentation of this article. The first author would like to thank Prof. Xindan Li, Dean of the School of Management and Engineering of Nanjing University, for providing excellent research facilities. The second author was supported by NSFC 71173098 .

Received: 11 March 2014 Accepted: 6 June 2014 Published: 22 Jul 2014

\section{References}

1. Peng, JW, Yao, JC: A new hybrid-extragradient method for generalized mixed equilibrium problems and fixed point problems and variational inequality problems. Taiwan. J. Math. 12(6), 1401-1432 (2008)

2. Al-Mazrooei, AE, Latif, A, Yao, JC: Solving generalized mixed equilibria, variational inequalities,and constrained convex minimization. Abstr. Appl. Anal. 2014, Article ID 587865 (2014)

3. Ansari, QH, Schaible, S, Yao, JC: The system of generalized vector equilibrium problems with applications. J. Glob. Optim. 22, 3-16 (2002)

4. Ceng, LC, Hu, HY, Wong, MM: Strong and weak convergence theorems for generalized mixed equilibrium problem with perturbation and fixed pointed problem of infinitely many nonexpansive mappings. Taiwan. J. Math. 15(3), 1341-1367 (2011)

5. Ceng, LC, Guu, SM, Yao, JC: Hybrid iterative method for finding common solutions of generalized mixed equilibrium and fixed point problems. Fixed Point Theory Appl. 2012, Article ID 92 (2012)

6. Ceng, LC, Ansari, QH, Schaible, S, Yao, JC: Iterative methods for generalized equilibrium problems, systems of general generalized equilibrium problems and fixed point problems for nonexpansive mappings in Hilbert spaces. Fixed Point Theory 12(2), 293-308 (2011)

7. Yamada, I: The hybrid steepest-descent method for the variational inequality problems over the intersection of the fixed point sets of nonexpansive mappings. In: Batnariu, D, Censor, Y, Reich, S (eds.) Inherently Parallel Algorithms in Feasibility and Optimization and Their Applications, pp. 473-504. North-Holland, Amsterdam (2001)

8. Colao, V, Marino, G, Xu, HK: An iterative method for finding common solutions of equilibrium and fixed point problems. J. Math. Anal. Appl. 344, 340-352 (2008)

9. Korpelevič, GM: An extragradient method for finding saddle points and for other problems. Èkon. Mat. Metody 12(4), 747-756 (1976)

10. Xu, HK: Averaged mappings and the gradient-projection algorithm. J. Optim. Theory Appl. 150(2), 360-378 (2011)

11. Ceng, LC, Yao, JC: A hybrid iterative scheme for mixed equilibrium problems and fixed point problems. J. Comput. Appl. Math. 214, 186-201 (2008)

12. Takahashi, S, Takahashi, W: Strong convergence theorem for a generalized equilibrium problem and a nonexpansive mapping in a Hilbert space. Nonlinear Anal. 69, 1025-1033 (2008)

13. Chang, SS, Joseph Lee, HW, Chan, CK: A new method for solving equilibrium problem, fixed point problem and variational inequality problem with application to optimization. Nonlinear Anal. 70, 3307-3319 (2009)

14. Qin, X, Shang, M, Su, Y: A general iterative method for equilibrium problem and fixed point problem in Hilbert spaces. Nonlinear Anal. 69, 3897-3909 (2008)

15. Yao, Y, Cho, YJ, Liou, YC: Iterative algorithms for hierarchical fixed points problems and variational inequalities. Math. Comput. Model. 52(9-10), 1697-1705 (2010)

16. Mainge, PE, Moudafi, A: Strong convergence of an iterative method for hierarchical fixed-point problems. Pac J. Optim. 3(3), 529-538 (2007)

17. Moudafi, A: Krasnoselski-Mann iteration for hierarchical fixed-point problems. Inverse Probl. 23(4), 1635-1640 (2007)

18. Cianciaruso, F, Marino, G, Muglia, L, Yao, Y: On a two-step algorithm for hierarchical fixed point problems and variational inequalities. J. Inequal. Appl. 2009, Article ID 208692 (2009)

19. Gu, G, Wang, S, Cho, YJ: Strong convergence algorithms for hierarchical fixed points problems and variational inequalities. J. Appl. Math. 2011, Article ID 164978 (2011)

20. Marino, G, Xu, HK: Explicit hierarchical fixed point approach to variational inequalities. J. Optim. Theory Appl. 149(1)، 61-78 (2011)

21. Ceng, LC, Ansari, QH, Yao, JC: Some iterative methods for finding fixed points and for solving constrained convex minimization problems. Nonlinear Anal. 74, 5286-5302 (2011) 
22. Ceng, LC, Al-Mezel, SA, Latif, A: Hybrid viscosity approaches to general systems of variational inequalities with hierarchical fixed point problem constraints in Banach spaces. Abstr. Appl. Anal. 2014, Article ID 945985 (2014)

23. Bnouhachem, A: A modified projection method for a common solution of a system of variational inequalities, a split equilibrium problem and a hierarchical fixed-point problem. Fixed Point Theory Appl. 2014, Article ID 22 (2014)

24. Ceng, LC, Ansari, QH, Yao, JC: Iterative methods for triple hierarchical variational inequalities in Hilbert spaces. J. Optim. Theory Appl. 151, 489-512 (2011)

25. Tian, M: A general iterative algorithm for nonexpansive mappings in Hilbert spaces. Nonlinear Anal. 73, 689-694 (2010)

26. Geobel, K, Kirk, WA: Topics in Metric Fixed Point Theory. Stud. Adv. Math., vol. 28. Cambridge University Press, Cambridge (1990)

27. Suzuki, N: Moudafi's viscosity approximations with Meir-Keeler contractions. J. Math. Anal. Appl. 325, 342-352 (2007)

28. Xu, HK: Iterative algorithms for nonlinear operators. J. Lond. Math. Soc. 66, 240-256 (2002)

29. Acedo, GL, Xu, HK: Iterative methods for strictly pseudo-contractions in Hilbert space. Nonlinear Anal. 67, 2258-2271 (2007)

30. Wang, Y, Xu, W: Strong convergence of a modified iterative algorithm for hierarchical fixed point problems and variational inequalities. Fixed Point Theory Appl. 2013, Article ID 121 (2013)

31. Rockafellar, RT: On the maximality of sums nonlinear monotone operators. Trans. Am. Math. Soc. 149, 75-88 (1970)

32. Ansari, QH, Lalitha, CS, Mehta, M: Generalized Convexity, Nonsmooth Variational Inequalities and Nonsmooth Optimization. Taylor \& Francis, London (2013)

33. Ansari, QH, Yao, JC: Systems of generalized variational inequalities and their applications. Appl. Anal. 76, 203-217 (2000)

34. Ceng, LC, Wang, CY, Yao, JC: Strong convergence theorems by a relaxed extragradient method for a general system of variational inequalities. Math. Methods Oper. Res. 67, 375-390 (2008)

35. Ceng, LC, Al-Mezel, SA, Ansari, QH: Implicit and explicit iterative methods for systems of variational inequalities and zeros of accretive operators. Abstr. Appl. Anal. 2013, Article ID 631382 (2013)

36. Cianciaruso, F, Marino, G, Muglia, L, Yao, Y: A hybrid projection algorithm for finding solutions of mixed equilibrium problem and variational inequality problem. Fixed Point Theory Appl. 2010, Article ID 383740 (2010)

37. Crombez, G: A hierarchical presentation of operators with fixed points on Hilbert spaces. Numer. Funct. Anal. Optim. 27, 259-277 (2006)

38. Latif, A, Ceng, LC, Ansari, QH: Multi-step hybrid viscosity method for systems of variational inequalities defined over sets of solutions of equilibrium problem and fixed point problems. Fixed Point Theory Appl. 2012, Article ID 186 (2012)

39. Marino, G, Muglia, L, Yao, Y: Viscosity methods for common solutions of equilibrium and variational inequality problems via multi-step iterative algorithms and common fixed points. Nonlinear Anal. 75, 1787-1798 (2012)

40. Peng, JW, Yao, JC: Strong convergence theorems of iterative scheme based on the extragradient method for mixed equilibrium problems and fixed point problems. Math. Comput. Model. 49, 1816-1828 (2009)

41. Verma, RU: General convergence analysis for two-step projection methods and applications to variational problems Appl. Math. Lett. 18, 1286-1292 (2005)

10.1186/1687-1812-2014-155

Cite this article as: Bnouhachem and Chen: An iterative method for a common solution of generalized mixed equilibrium problems, variational inequalities, and hierarchical fixed point problems. Fixed Point Theory and Applications 2014, 2014:155

\section{Submit your manuscript to a SpringerOpen ${ }^{\circ}$ journal and benefit from:}

- Convenient online submission

- Rigorous peer review

- Immediate publication on acceptance

- Open access: articles freely available online

- High visibility within the field

- Retaining the copyright to your article 